NBSIR 81-2398

\title{
Investigation of the Two-Dimensional Shape of Ion-Implanted Regions
}

U.S. DEPARTMENT OF COMMERCE

National Bureau of Standards

National Engineering Laboratory

Center for Electronics and Electrical Engineering

Semiconductor Devices and Circuits Division

Washington, DC 20234

and

Sandia National Laboratories

(U.S. Department of Energy Facility)

Albuquerque, NM 87185

December 1981

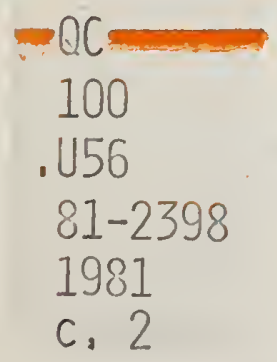

Naval Ocean Systems Center

San Diego, CA 92152 


\section{INVESTIGATION OF THE TWO-DIMENSIONAL SHAPE OF ION-IMPLANTED REGIONS}

U.S. DEPARTMENT OF COMMERCE

National Bureau of Standards

National Engineering Laboratory

Center for Electronics and Electrical Engineering

Semiconductor Devices and Circuits Division

Washington, DC 20234

and D. R. Myers

Sandia National Laboratories

(U.S. Department of Energy Facility)

Albuquerque, NM 87185

December 1981

Prepared for

Naval Ocean Systems Center

San Diego, CA 92152

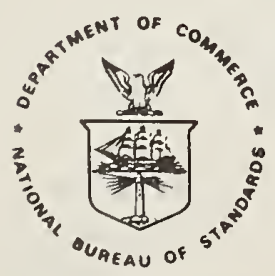

U.S. DEPARTMENT OF COMMERCE, Malcolm Baldrige, Secretary NATIONAL BUREAU OF STANDARDS, Ernest Ambler, Director 


$$
\text { 4. }
$$

t.


3. Review of Implantation Modeling . . . . . . . . . . . . . . . 6

A) Fundamentals of Ion Penetration Theory . . . . . . . . . . 6

i) Introduction . . . . . . . . . . . . . . . . . 6

ii) Interaction Mechanisms . - . - . . . . . . . . . 6

B) Ion Range Theories in One Dimension for Amorphous Targets . . 8

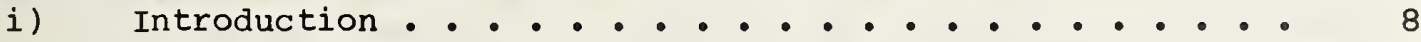

ii) Analytic Codes . . . . . . . . . . . . . . . . 12

iii) Monte-Carlo Codes . . . . . . . . . . . . . . . 14

iv) Comparison to Experiments . • . • . . . . . . . . . . 14

C) Ion Range Theories for Point Sources . . . . . . . . . . 18

i) Introduction . . . . . . . . . . . . . . . . . 18

ii) Analytic Theories . . . . . . . . . . . . . . 18

iil) Monte-Carlo Codes . . . . . . . . . . . . . . . 19

iv) Comparison of Predictions . . . . . . . . . . . . . 19

D) Scattering Under a Rectangular Mask Edge . . . . . . . . ..$\quad 21$

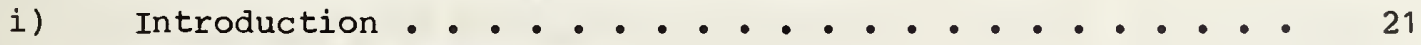

ii) Comparison of Theories . . . . . . . . . . . . . 21

E) Ion Channeling . . . . . . . . . . . . . . . . . . . 25

i) Introduction . . . . . . . . . . . . . . . . . . 25

ii) The Continuum Model of Lindhard . . . . . . . . . . 25

ii) The Effect of Amorphous Surface Layers . . . . . . . 28

iv) "Lateral Channeling" . . . . . . . . . . . . . . . 31

F) Electrical Activation of Arsenic-Implanted Silicon . . . . . . 31

4. Experimental . . . . . . . . . . . . . . . . . . . . 33

A) Sample Fabrication . . . . . . . . . . . . . . . . 33

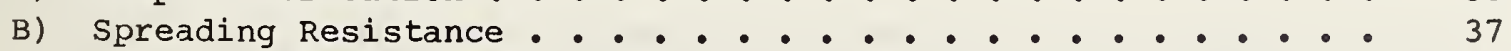

C) Etch Technique . . . . . . . . . . . . . . . . . . 41

i) Contrast Mechanism . . . . . . . . . . . . . 441

ii) Etch Data • . . . . . • . . . . . . . . . . . 41

iii) Interpretation and Problems with Etch Data . . . . . 45

D) Induced Current Techniques . . . . . . . . . . . . . . 50

i) Physics of Electron-Beam-Induced Currents . . . . . . 50

ii) EBIC Data . . . . . . . . . . . . . . . 51

iii) Interpretation of EBIC Data . . . . . . . . . . 51

5. Conclusions • • • • • • • • • • • • • • • • . . . . 55

References . . • • . . . . . . . . . . . . . . . . . 59 
3.1 Plots of the $f(\phi)$ function as given as eq (3.10) for several representative potentials parameterized by the values of $\lambda, m$, and $\mathrm{q}$ as given in table 3.1 .

(a) Results for the Thomas-Fermi (Lindhard), the Chu-Murley (Wilson), the Moliere, the Lenz-Jensen, and the Bohr potentials

(b) The function $S_{n}(\varepsilon)$ for figure $3.1 \mathrm{a}$, along with values of electronic stepping power common to typical implantations . .

3.2 Predictions of the Winterbon amorphous target code with several choices of the potential for the case of a 150-keV implant of arsenic into silicon at a dose of $5 \times 10^{15} \mathrm{~cm}^{-2} . . .$. . .

3.3 Comparison of the TRIM (Monte-Carlo) code and the Winterbon amorphous target code for 150-kev implant of arsenic into silicon at a dose of $1 \times 10^{15} \mathrm{~cm}^{-2}$.............

3.4 Comparison of profiles for 150-keV arsenic implanted into sili-

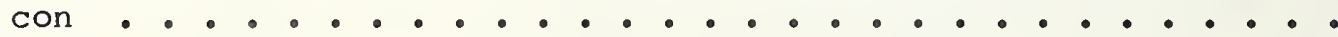

3.5 Predicted ion density distributions resulting from a point source of $75-\mathrm{keV}$ arsenic ions incident on amorphous silicon at o-deg incidence . . . . . . . . . . . . . . . . .

3.6 Predicted ion density distributions resulting from 75-kev arsenic implantation into amorphous silicon partially covered by a rectangular mask.

(a) The density contour after Furukawa using a product of a complementary error function vertically and a Gaussian horizontally

(b) The density contour after Ryssell using a product of a Pearson distribution vertically and a Gaussian horizontally . .

(c) The density contour calculated by the TRIM code using the Moliere potential

3.7 The Lindhard string model for the interaction of an incident ion traveling along a channel ...............

3.8 Variation in probability of ion channeling versus tilt angle, and step function approximation . . . . . . . . . . .

4.1 Schematic diagram of the resist structure used to mask the implants......................

4.2 SEM micrograph of the photoresist structure used to mask the implants . . . . . . . . . . . . . . . . . . 
4.3 SEM micrograph of the photoresist structure used to mask the implants . . . . . . . . . . . . . . . . . . .

4.4 Schematic diagram of an etch step at the corner of the grating pattern ..................... . . . . 38

4.5 SEM micrograph of an etch step . . . . . . . . . . . 39

4.6 Comparison of a typical spreading resistance profile with the predictions of the SUPREM model using joined half-Gaussians and with the amorphous target code . . . . . . . . . . .

4.7 Schematic diagram of the electron-beam-sample interaction near an etch step..........................

4.8 Secondary electron picture of a cleaved and etched sample tilted 0.5 deg to normal (at $75 \mathrm{keV}$ and 0 -deg tilt) .....

4.9 Secondary electron picture of a cleaved and etched sample tilted 0.5 deg to normal (at $150 \mathrm{keV}$ and 7-deg tilt) . . . .

4.10 Secondary electron pictures of cleaved and etched samples tilted 0.5 deg to normal (at $75 \mathrm{keV}$ and 7 -deg tilt).

(a) Annealed at $1000^{\circ} \mathrm{C}$ for $30 \mathrm{~min}$. . . . . . . 47

(b) Annealed at $1000^{\circ} \mathrm{C}$ for $60 \mathrm{~min}$.............. 48

4.11 Secondary electron picture of a cleaved and etched sample 0.5 deg to normal ...................

4.12 Composite secondary electron and EBIC micrograph . . . . . . 52

4.13 Higher magnification of figure 4.12 . . . . . . . . 53

4.14 Deflection modulation EBIC picture . . . . . . . . 54

5.1 Comparison of junction depths determined by junction etch and by the as-implanted joined half-Gaussian model used in SUPREM for a sample implanted at $75 \mathrm{keV}$ and annealed at $600^{\circ} \mathrm{C}$. . . . .

5.2 Comparison of junction depths determined by junction etch and by several theoretical models for a sample implanted at $150 \mathrm{keV}$ and annealed at $600^{\circ} \mathrm{C}$. . . . . . . . . . . . . .

5.3 Comparison of junction depths determined by junction etch and by two models for a $5 \times 10^{15} \mathrm{~cm}^{-2} 150-\mathrm{keV}$ arsenic-implanted sample annealed at $1000^{\circ} \mathrm{C}$ for $30 \mathrm{~min}$. . . . . . . . . . . 


\section{List of Tables}

Page

3.1 Parameterization of the Cross Section in Terms of the Potential 9

3.2 Dechanneling of Arsenic Implants into (100) Silicon by Amorphous Surface Layers for 0 -Deg Incident Beam . . . . . . . . .

4.1 Comparison of Junction Depth Locations Obtained from Spreading Resistance and Stain Techniques .............. 
The work described in this report was sponsored by the Naval Ocean Systems Center (Contract \#MIPR N6600179MP00052) and the National Bureau of Standards.

A special acknowledgment is given to Dr. Isaac Lagnado of the Naval Ocean Systems Center for his insight and understanding of the need for the present investigation as well as his continued support and encouragement during the course of the work.

The Sandia National Laboratories (Contract DE-AC04-76-DP00789) provided computer facilities and time for the Monte-Carlo calculations of the twodimensional distributions. Dr. D. R. Myers was with the National Bureau of Standards when this project was initiated. 


\author{
Peter Roitman, John Albers, James R. Ehrstein \\ Semiconductor Devices and Circuits Division \\ National Bureau of Standards \\ Washington, D.C. 20234 \\ and \\ David R. Myers \\ Sandia National Laboratories \\ (U.S. Department of Energy Facility) \\ Albuquerque, NM 87185
}

\begin{abstract}
The two-dimensional shape of arsenic ion-implanted regions in single-crystal silicon was investigated both experimentally and theoretically. Experimentally, two techniques were shown to have the necessary submicron resolution: a junction etch process and an SEM-induced current collection method. A comparison of junction depths determined by the etch technique, the EBIC technique, and spreading resistance with the depths calculated using several amorphous target codes was made. For the case of low temperature $\left(600^{\circ} \mathrm{C}\right)$ anneals, the etch technique agrees very well with the junction depths predicted by the amorphous target code due to winterbon. The lateral junction locations obtained from the etch technique are in good agreement with the predictions of a twodimensional Monte-Carlo code (TRIM) which indicates that arsenic does not show any significant lateral scattering under mask edges. For the case of high temperature $\left(1000^{\circ} \mathrm{C}\right)$ anneals, the etch and EBIC techniques agree with each other, but show consistently deeper junction locations than does the spreading resistance technique. Comparison with arsenic-diffusion models shows that concentrationdependent effects are important. Presently available processing models do not appear to adequately forecast junction depths.
\end{abstract}

Key words: arsenic ion implantation; electron-beam-induced current; etching; integrated circuit processing; ion channeling; ion implantation; lateral profiles; scanning electron microscopy; silicon technology; spreading resistance; two-dimensional profiles. 


\section{INTRODUCTION}

Ion implantation is the preferred means of dopant introduction for semiconductor integrated circuit fabrication due to the reproducibility, lateral uniformity, dopant level control, and lateral registration which can be achieved. These features give ion implantation a significant advantage over diffusion technology in the fabrication of LSI devices; however, as device dimensions in integrated circuits are reduced for very high speed, very large scale integrated circuits, device performance will become increasinqly sensitive to the details of the depth distribution of dopants, and secondary processes characterized by the two-dimensional nature of implanted (and annealed) regions will become limiting factors in device performance. Despite the present recognition that dopant control in two dimensions is of critical importance, the processes that control the ultimate shape of the doped regions especially during implantation are not adequately understood.

To illustrate the increasing importance of two-dimensional dopant profile control, it is useful to examine the application of ion implantation to the fabrication of silicon enhancement-mode metal-oxide-semiconductor fieldeffect transistors (MOSFETs) for use in integrated circuits. In the late 1960s, typical variations in substrate background doping were 20 percent, leading to unacceptable variations in threshold voltage across an integrated circuit [1]. Implantation was then applied to control the doping in the channel [2]; and it was found that for shallow enough implants at low enough doses, the implanted dopants were always depleted for zero-bias conditions. For those implants, the threshold voltage was found to scale linearly with implanted dose $[1,2]$, and for those devices, implanted ions acted as a variation in channel doping level. Prior to the introduction of implantation, lateral diffusion under the gate during source and drain formation led to increased parasitic capacitances that reduced device operating speed [1]. In addition to allowing controlled variations in doping level, the introduction of implantation also allowed for the formation of self-aligned devices [3] that greatly reduced device parasitics, due to the reduced penetration of implanted dopants under a mask edge [4] compared to the extent of lateral diffusion typical in diffused devices. As device dimensions are reduced to produce faster and denser circuits, linear scaling requires that junction depths and lateral dimensions be decreased and dopant levels increased [5]. However, as the dose of the threshold adjustment implant is increased, all the implanted dopants are no longer totally depleted, and the threshold voltage no longer scales linearly with ion dose [6]. Of even greater importance is the fact that linear scaling is no longer sufficient to ensure proper device operation [7], as secondary effects such as short-channel effects [8], drain-induced barrier lowering [9], punch through [10], substrate reversebias sensitivity [7], subthreshold conduction [11], snap-back [12], and hot carrier effects [13] become the limiting factors in device performance. All of these effects are determined by the details of the electric field distribution determined by the two-dimensional interactions among the doped regions and thus demand control over not only the depth distribution of the implanted ions but their lateral spreading as well.

The importance of two-dimensional dopant profile control is not limited to silicon enchancement-mode MOSFETs. Dopant profile effects in two dimensions also control device operation in depletion-mode transistors in silicon or in 
gallium arsenide [14]. Finally, in silicon bipolar technologies the net dopant profile at the junction between the emitter and the active base controls transistor turn-on and turn-off characteristics by determining emitterbase capacitance [15] and such other parameters as base width, forward gain, and base spreading resistance [16]. Thus, control over dopant profiles resulting from implantation is of great importance for future device technologies. 


\section{ION CHANNELING DURING IMPLANTATION}

It has been long recognized that ion distributions resulting from implantation into initially crystalline targets are affected by ion channeling [17], in which ions are steered down axial lattice channels by correlated collisions with the ordered arrangement of lattice atoms. While this phenomenon is qualitatively understood on the basis of an analytic theory of Lindhard [18], the major advantage of this theory is that it scales implant ion mass, energy, and alignment to substrate crystallographic orientation in terms of a calculated critical angle for channeling. This angle varies from 2 to 7 deg for common industrial implantation [19], with the larger critical angles being found for implantations of heavier ions at low energies, as will be used to produce shallow doped regions for future devices. What is not generally understood, however, is that the critical angle so calculated corresponds only to the limits of applicability of the assumptions made in the theory and not to the actual limits of angular alignment over which substantial channeling can occur [20]. Studies of ion channeling during implantation $[19,21]$ indicate that channeling characteristics persist until ion beam alignment to the crystallographic orientations exceeds twice the critical angle. It is important to note that the angle between the ion beam and the nearest low index crystallographic direction required to minimize unintentional channeling can easily exceed the 7-deg tilt angle commonly used for industrial implantation and that a substantial amount of ion channeling can occur at 7-deg tilt angles for implantation of heavy ions at low energy. As an example, 53.5 percent of $75-\mathrm{keV}$ arsenic ions implanted into (111) silicon at a 7-deg tilt angle penetrate deeper than the LSS Gaussian distribution $[19,21]$, with even greater amounts of ion channeling occurring for arsenic implantations at lower energies for nominal "random equivalent" implantation.

In considering implantation for device structures, several important factors must be considered. Junctions between doped regions occur at impurity densities typically four or more orders of magnitude lower than the peak dopant densities in the doped regions; thus, the thirty or more times greater penetration of channeled heavy ions $[19,21]$ can lead to junction locations removed from that predicted on the basis of range theories for amorphous targets before implantation-induced damage reduces the possibility of ion channeling. In addition, since ion trajectories in crystalline substrates are determined by the conditions under which the ion enters the crystalline region [18-20], the presence of an amorphous covering layer is not always sufficient to eliminate unintentional channeling in underlying crystalline substrates [22]. The presence of an amorphous surface layer has been shown to reduce the extent of ion penetration for directly aligned implants [23]. It has also been found, however, that for certain implant conditions (ion species, implant energy, substrate orientation, and amorphous surface layer thickness) the angular divergence introduced into the ion beam by random collisions with atoms in the amorphous surface layer can compensate for the effects of substrate tilt angle and actually increase the extent of unintentional channeling by orienting the ions back toward the major (low index) crystallographic directions [24]. 
From the above discussion, several important facts should be noted:

(1) Unintentional channeling strongly affects the ultimate depth distribution of dopants resulting from implantation.

(2) The extent of unintentional channeling is commonly underestimated for typical device implantations.

(3) Neither a 7-deg tilt angle nor a surface layer of silicon dioxide is sufficient to prevent unintentional channeling during implantation.

(4) No adequate theoretical treatment of the effects of unintentional channeling on the two-dimensional penetration of implanted dopants exists.

Because of the increasing importance of lateral control of implanted dopant profiles, an empirical study of the effects of unintentional channeling on the two-dimensional profile of implanted regions has been performed to determine data for device designers, as well as to generate a data base on the systematics of lateral penetration during implantation so that physical understanding can be developed. This study is especially timely as these factors control the depths of the $0.2-\mu \mathrm{m}$ deep junctions required by scaling laws for 1.0- $\mu \mathrm{m}$ lateral surface dimension geometries [25]. 


\section{REVIEW OF IMPLANTATION MODELING}

\section{A) Fundamentals of Ion Penetration Theory}

\section{i) Introduction}

When an energetic ion enters a solid, it undergoes collisions with the lattice core ions and electrons. These collisions are responsible for slowing down the incident ion and eventually bringing it to rest at some vector distance from the original point of entry. In addition to the stopping of the incident ion, several other phenomena are possible. If the target is crystalline, the incident ion may destroy the regular long-range order of the crystal by displacing the lattice ions from their equilibrium positions. This phenomenon, referred to as radiation damage, usually proceeds from isolated damage regions at low incident ion fluences to overlapping damage regions, i.e., complete amorphization, at high incident ion fluences. The onset of amorphization depends upon the ratio of the masses of the incident ion and the target ion. Generally, lower fluences are needed for amorphization when the ratio is larger than unity, and vice versa.

As the effects of lattice damage (and the subsequent incomplete activation of the implanted dopant atoms) must be eliminated by either thermal or laser annealing, the principal concern of ion implantation modeling is the prediction of the spatial distribution of the implanted dopant atoms and their subsequent redistribution and activation by the annealing process.

This section of the report will be concerned with the development of a physical understanding of the lateral spreading process and the application of predictive models.

To develop this necessary physical understanding, the basic mechanisms by which ions come to rest in a solid will be briefly reviewed. It will be seen that there are a number of different models for the ion-target interaction, and these will be evaluated on the basis of their predictions of the depth distribution of impurities resulting from implantation into bare silicon surfaces. Both analytic and statistical (Monte-Carlo) methods will be examined. The approach will then be generalized to the case of two-dimensional distributions, where it will be seen that the Monte-Carlo methods offer significant advantages. The effects of crystalline order on ion trajectories will then be discussed, where it will be shown that ion channeling affects the vertical, but not the lateral, dimensions. Finally, the modification of the implanted profiles by diffusion during annealing will be briefly discussed.

\section{ii) Interaction Mechanisms}

As indicated above, the interaction between the incident energetic ion and the target atoms is central to the prediction and understanding of ion ranges in solids. Ions lose energy in a solid by two distinct mechanisms: through collisions with the screened nuclei of the target atoms (nuclear stopping), and also through interaction with the electrons surrounding the nuclei of the target atoms (electronic stopping). In early formulations of ion range theories, the two processes were viewed as independent processes; but more recent 
formulations allow for correlation of the two processes. As excellent reviews of the subject exist (e.g., [26]), only the salient features will be summarized here to provide a background for the range calculations and to interpret the experimental results.

Nuclear stopping arises from the interaction between the incident ion and the core ions in the solid. This process is viewed as being a sequence of twobody nuclear encounters; i.e., the incident ion scatters off one core ion at a time. Further, for a given ion-ion potential, the dynamics of the collision process is formulated in terms of classical two-body scattering theory. Also, because of the nature of the ion-atom scattering, it is this portion of the interaction between the incident ion and the solid which is primarily responsible for directional effects, i.e., the change of direction of motion of the incident ion from that of the incident beam. These nuclear encounters are responsible not only for the scattering of ions under a mask edge for implantation into an amorphous target, but also for the correlated collisions that produce ion channeling in a crystalline target.

Ions moving through a solid lose energy to electrons as well. Starting from two different physical models, either Firsov [27] or Lindhard and winther [28] have derived that electronic stopping should be directly proportional to the ion's velocity, similar to a viscous drag force. Also similar to a viscous drag force, electronic stopping is usually viewed as having no effect on the particle's direction.

The relative importance of nuclear and electronic stopping in determining ion penetration depths is influenced both by the ion's incident energy and by crystal lattice effects. For ions that follow a trajectory in which the substrate atoms appear to be randomly arranged, the lower the ion's incident energy, the more important nuclear stopping will become in determining the ultimate ion range. Ions that channel, however, do not approach the target atoms closely enough to lose appreciable energy in nuclear collisions, and thus lose most of their energy by electronic stopping. For heavy ions at low energies (such as those studied here), the excess penetration of the ions that channel over those that follow a random trajectory is extremely important in establishing vertical junction depth.

Thus far, nothing has been said about the specific form of the two-body potential which is responsible for the incident-ion core-ion scattering process. Historically, the work of Lindhard et al. [29] made use of a screened coulomb potential. They have shown for several forms of this potential that the energy and angular dependence of the cross section for ion scattering from a target atom may be expressed as

$$
d \sigma=\pi a^{2} \frac{d \phi}{\phi^{2}} f(\phi),
$$

where the function $f(\phi)$ depends upon the screening length, a; while the variable $\phi$ is an energy transfer variable defined by

$$
\phi^{2}=\varepsilon^{2} \mathrm{~T} /(\gamma E),
$$

where $T$ is the energy transferred in the collision process, $Y E$ is the maximum energy transfer, and $\varepsilon$ is a dimensionless energy 


$$
\varepsilon=\left(\frac{M_{2} E}{M_{1}+M_{2}}\right)\left(\frac{z_{1} z_{2} e^{2}}{a 4 \pi \varepsilon_{0}}\right)^{-1},
$$

where $M_{1}=$ ion mass

$M_{2}=$ target atom mass

$z_{1}, z_{2}=$ atomic numbers of the ion and target atoms, respectively, and $\mathrm{e}=$ electronic charge in MKS units.

For purposes of analysis and calculation, the function $f(\phi)$ may be expressed in the form

$$
f(\phi)=\lambda \phi^{1-2 m}\left(1+\left(2 \lambda \phi^{2-2 m}\right)^{q}\right)^{-1 / q},
$$

which leads to a nuclear stopping power

$$
S_{n}(\phi)=[2 \phi(2-2)]^{-1} \int_{0}^{2 \phi} f\left(1-u_{q}\right)^{-1} d u
$$

This parameterization has been used by Winterbon to generate a code to predict ion ranges in amorphous targets [30], and in that work the parameters were also calculated for a number of potentials other than the Thomas-Fermi form. A number of experimental studies (e.g•, [31]) have demonstrated that the Thomas-Fermi potential overestimates nuclear stopping at low energies ( $\varepsilon$ from eq (3.3)<0.1). Wilson, Haggmark, and Biersack (WHB) [32] have calculated nuclear stopping, power, range, and straggling in the low-energy region for 14 diatomic interactions and have generated an average potential as a "universal" approximation. This work also demonstrated that, of the previously existing expressions for the interatomic potential, the Moliere potential was closest to the WHB average potential. The WHB average potential has been parameterized by Chu et al. [31] in a form appropriate for the Winterbon code and has been found to yield results in excellent agreement with experiment. Table 3.1 presents the choices of the parameters $\lambda, m$, and $q$ which correspond to several choices of potential. Also, for purposes of comparison, the function $S_{n}(\varepsilon)$ is plotted in figure 3.1. From this plot, it can be seen that the various choices of the parameters represent various degrees of potential range and strength, and thus each potential would predict different ion ranges and lateral spreading for a given particular implant. Also shown are representative particular implant values of electronic stopping power.

B) Ion Range Theories in One Dimension for Amorphous Targets

\section{i) Introduction}

Collisions with substrate atoms necessarily produce three-dimensional scattering of the implanted ions; however, implantation into large unmasked surface areas produces an overlap of large numbers of ion trajectories, thus resulting in a laterally uniform ion density distribution. When the distribution of implanted ions varies only with depth into the solid, the distribution can be more easily determined than a two-dimensional distribution, since most experimental techniques sample an area large compared to the lateral 
TABLE 3.1 - Parameterization of the Cross Section in Terms of the Potential. POTENTIAL $\lambda$

m

q

$\begin{array}{llll}\text { Thomas-Fermi (Lindhard) } & 1.309 & 0.33333 & 0.666666 \\ \text { Chu-Murley (WHB) } & 2.542 & 0.25 & 0.50 \\ \text { Moliere } & 3.071 & 0.2157 & 0.5303 \\ \text { Lenz-Jensen } & 2.917 & 0.1909 & 0.5116 \\ \text { Bohr } & 2.37 & 0.1032 & 0.5696 \\ \text { Kalbitzer (Wilson) } & 2.54 & 0.26 & 0.475\end{array}$

The cross section as given by eq (3.1) is related to the potential listed by means of the appropriate choice of the parameters $\lambda, \mathrm{m}$, and $\mathrm{q}$ as listed. 


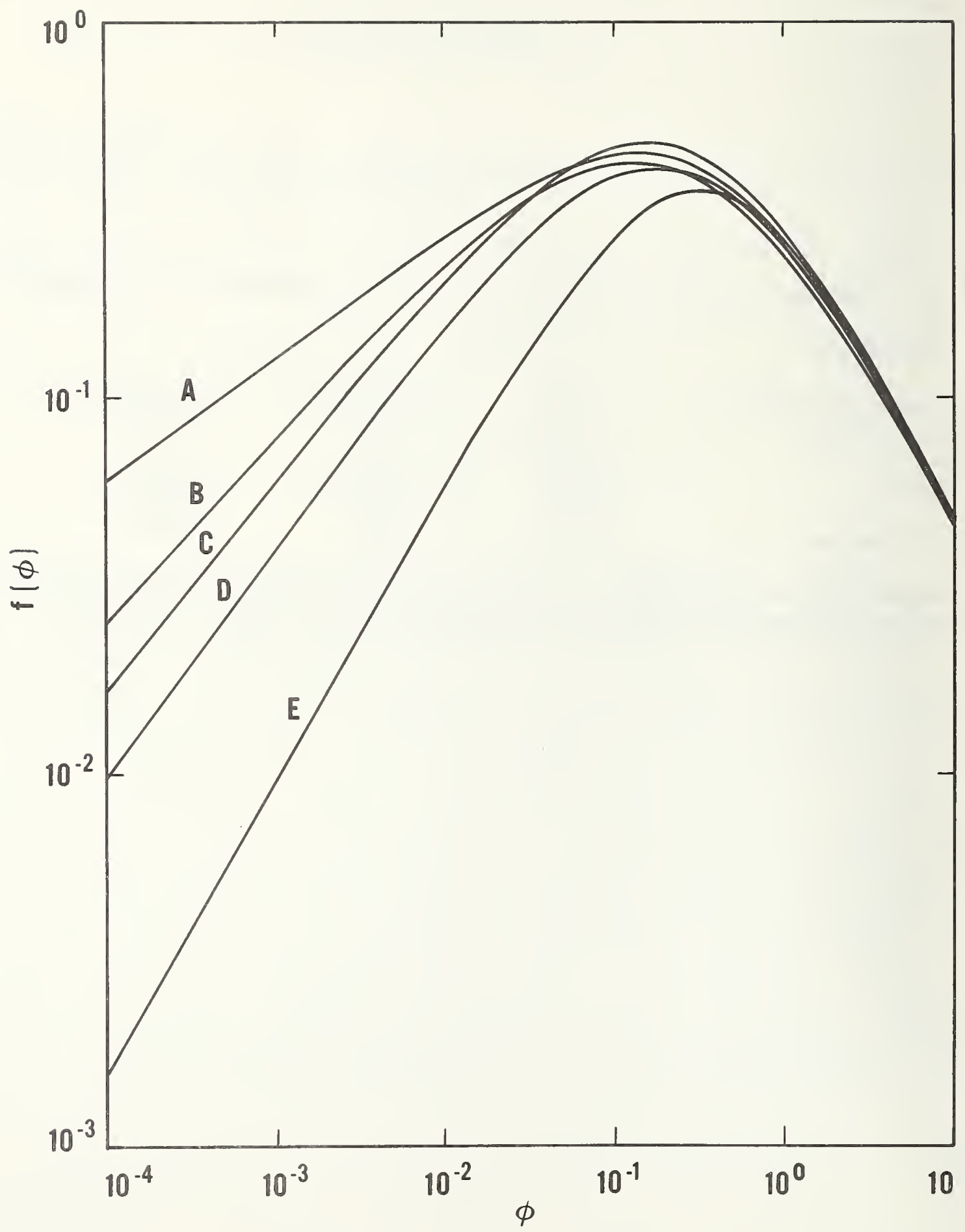

(a) Results for the Thomas-Fermi (Lindhard) (A), the Chu-Murley (WHB) (B), the Moliere (C), the Lenz-Jensen (D), and the Bohr (E) potentials.

Figure 3.1 Plots of the $f(\phi)$ function as given as eq (3.10) for several representative potentials parameterized by the values of $\lambda, m$, and $q$ as given in table 3.1. 


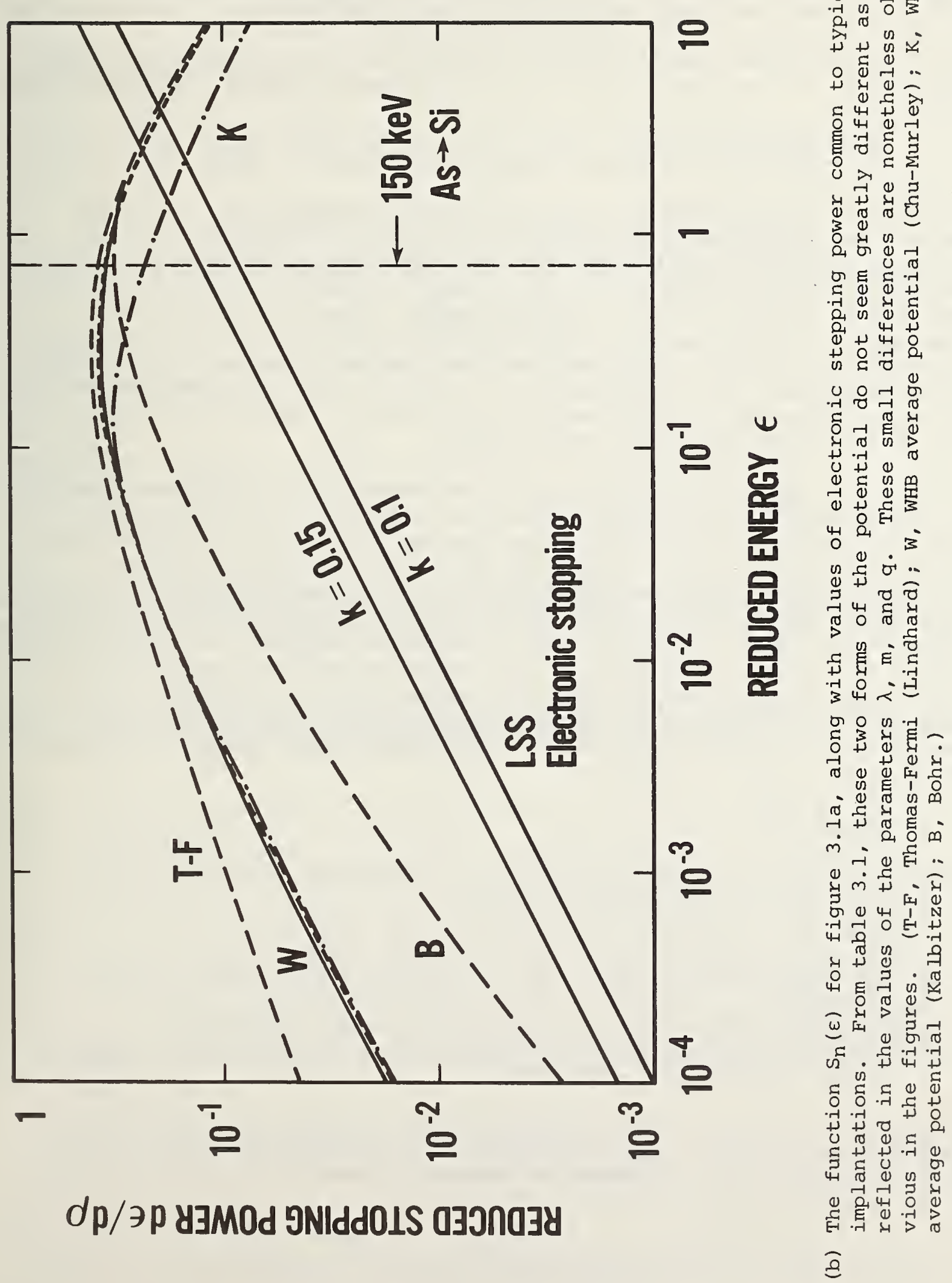


spreading of an ion beam. These one-dimensional distributions thus provide an important check of computational and analytic procedures and provide a basis for the two-dimensional studies to be described later.

Sanders [33] has shown that the spatial distribution in one dimension of ions implanted into an amorphous target follows the linear Boltzmann equation of the form

$$
\left|\frac{V}{V}\right| \frac{\partial F(X, V)}{\partial X}=N \int d \sigma\left\{F(X, V)-F\left(X, V^{\prime}\right)-\varepsilon F\left(X, V^{\prime \prime}\right)\right\}+\frac{S}{V} \frac{\partial F}{\partial V},
$$

where $F(X, V)$ is the distribution function of the implanted ions which depends upon the vector position, $\mathrm{x}$, and upon the particle velocity, $\mathrm{V}$; $\mathrm{N}$ is the number density of target atoms; do is the scattering cross section which depends upon the potential between the incident ion and the core-ions of the target; and $\mathrm{S}_{e}$ is the electronic stopping power. Central to the use of this equation is the assumption that the collisions between the incident ion and the lattice core-ions are binary and that the collision is of effectively zero-time duration. The justification of the use of the linear Boltzmann equation and a derivation have been given by Hynes and Deutch [34] following the previous work of Albers and Deutch [35].

The linear Boltzmann equation describing the distribution of implanted ions is an intergo-differential equation which is difficult to solve in general. Instead of solving for the distribution function directly, the theories presented by Lindhard, Scharff, and Schiфtt [29] and by Winterbon, Sigmund, and Sanders [36] rely upon the solution of a set of coupled equations for the moments of the distribution. These methods will be referred to as analytic solutions, since they attempt to solve the Boltzmann equation for the form of the range distribution directly. An alternative method (as exemplified by the TRIM code [37]) relies on Monte-Carlo methods [38] to construct the ion distribution by solving the equations of motion for a single ion and then repeating the calculation for a statistically significant number of ions with randomly chosen initial collisions.

\section{ii) Analytic Codes}

In this study, the amorphous target range code generated by Winterbon [39] was used because of the following advantages:

1. The code is available from its author on request.

2. The code allows the option of correlating nuclear and electronic stopping.

3. The code allows the use of different ion-atom potentials.

4. The code solves the moment equations directly rather than iteratively for both $\mathrm{x}$ - and $\mathrm{y}$-directions.

In general, the moments of the distribution (in one dimension) are given by the equation for a point or plane-sources and a subsequent expansion of the angular dependence of the equation in Legendre polynomials. The first condition for the plane source reduces eq (3.1) to

$$
-\cos \theta \frac{\partial F}{\partial X}=S_{e} \frac{\partial F}{\partial E}+M \int d \sigma\left\{F(X, E, \theta)-F\left(X, E^{\prime} \theta\right)-\varepsilon F\left(X, E^{\prime \prime}, \theta^{\prime \prime}\right)\right\},
$$


while the Legendre polynomial expansion as given by

$$
F(\cos \theta)=\sum_{\ell}(2 \ell+1) F_{\ell} P_{\ell},
$$

leads to a further reduction as

$$
\begin{aligned}
-\ell \frac{\partial F_{\ell-1}}{\partial X} & -(\ell+1) \frac{\partial F_{\ell+1}}{\partial X}=(2 \ell+1) S_{e} \frac{\frac{\partial F}{e}}{\partial E}+(2 \ell+1) N \int f \sigma\left\{F_{e}(X, E)\right. \\
& \left.-P_{\ell}\left(\cos \phi^{\prime}\right) F_{\ell}\left(X, E^{\prime}\right)-E P_{\ell}\left(\cos \phi^{\prime \prime}\right) F_{\ell}\left(S, E^{\prime \prime}\right)\right\} .
\end{aligned}
$$

Introducing the moment equation, which in the present case, takes the form

$$
F_{\ell}^{n}=\int x^{n} F_{\ell}(x) d x,
$$

leads to the equation

$$
\begin{aligned}
\frac{n\left(\ell F_{\ell-1}^{n-1}+(\ell+1) F_{\ell+1}^{n-1}\right)}{2 \ell+1} & =S_{e} \frac{\partial F_{\ell}^{n}+N}{\partial E} \int d \sigma\left\{F_{\ell}^{n}(E)-P_{\ell}\left(\cos ^{\prime}\right) F_{\ell}^{n}\left(E^{\prime}\right)\right. \\
& \left.-\xi P_{\ell}\left(\cos \theta^{\prime \prime}\right) F_{\ell}^{n}\left(E^{\prime \prime}\right)\right\} .
\end{aligned}
$$

This equation represents a recursion relation, in an integro-differential equation form, for the Legendre expansion coefficients for the moments of the distribution.

Without going into the details of the analysis and the accompanying code, the version of the Winterbon program used here solves the system of equations for the moments by means of a spline routine.

Once the moments have been calculated from the code, construction of the distribution from the moments is required. Unfortunately, this construction is not necessarily unique. The specific technique used most frequently in the construction of the distribution from a limited number of moments (four in this case) is due to Pearson (e.g., [40]). The input information in this scheme for the construction of the distribution are the mean (first moment relative to the surface), the standard deviation (the second moment relative to the surface), the skewness (related to the third central moment), and the kurtosis (related to the fourth central moment). The mean and standard deviation are given by eq (3.6) for the cases of $n=1$ and $n=2$, respectively. The skewness and the kurtosis are defined relative to the mean and the standard deviation by

$$
\text { skewness }=\frac{\left\langle(\mathrm{X}-\langle\mathrm{X}\rangle)^{3}\right\rangle}{(\text { s.tandard deviation })^{3}} \text {, }
$$

and

$$
\text { kurtosis }=\frac{\left\langle(x-\langle x\rangle)^{4}\right\rangle}{(\text { standard deviation })^{4}} \text {, }
$$


respectively. Depending upon the values of the skewness and the kurtosis, several forms of the resulting distribution may arise. These are usually referred to by the appropriate Pearson type.

It should be noted that this scheme, the Pearson scheme, is not the only scheme for the construction of the distribution from the moments. There are a number of other schemes which might be used. However, in this report, attention is primarily focused upon the Pearson systems.

The results of the winterbon code are sensitive not only to the choice of the potential, but also to the inclusion of correlated electronic scattering (through an impact-parameter dependent electronic coupling). Figure 3.2 represents the results of the calculation of arsenic implanted into silicon at $150 \mathrm{keV}$ at a fluence of $5 \times 10^{15} \mathrm{~cm}^{-2}$ for several choices of the potential. It can be seen that the potentials predict approximately the same modal depths but significantly different distributions, including peak densities and junction depths.

\section{iii) Monte-Carlo Code}

An alternative method which has been used in the calculation of ion ranges makes use of the Monte-Carlo technique [38]. The version of this method employed in this study is due to Biersack and Haggmark, the TRIM (Transport of Ions in Matter) code [37]. The central point of the Monte-Carlo technique which TRIM uses is not to solve an equation for the distribution for the implanted ions but rather to consider the details of the motion of individual ions as they travel into the target. For a given form of the scattering potential, the motion of individual incident ions is calculated until the energy falls below a predetermined limit at which point the ion is considered to have come to rest. The histories of a large number of incident ions (usually at least 1,000 ) are used to construct histograms of the number of ions which have come to rest in given depth intervals from the surface. These histograms represent the Monte-Carlo predictions of the range distributions.

The version of the TRIM code used here employs the Moliere potential previously described with uncorrelated nuclear and electronic stopping. The results of this code for the case of $150 \mathrm{keV}$ arsenic implanted into silicon are presented in fiqure 3.3, where they are contrasted with the results of the Winterbon code run for identical conditions. As can be seen from the figure, the two techniques yield very similar results for the same potential, thereby providing confidence in the numerical methods used for each technique.

\section{iv) Comparison to Experiments}

In figure 3.4, experimental results obtained by secondary ion mass spectrometry (SIMS) for a $150-\mathrm{keV}$ arsenic implant at a fluence of $5 \times 10^{15} \mathrm{~cm}^{-2}$ into a bare silicon surface are compared to the predictions of both the analytic and the Monte-Carlo codes.

In this figure, the results of the Monte-Carlo code have been left as histograms to help distinguish the various curves. As seen in this figure, both the analytic and the Monte-Carlo methods produce good agreement on the modal depth and peak density, as would be expected from figure 3.1. Both calcula- 


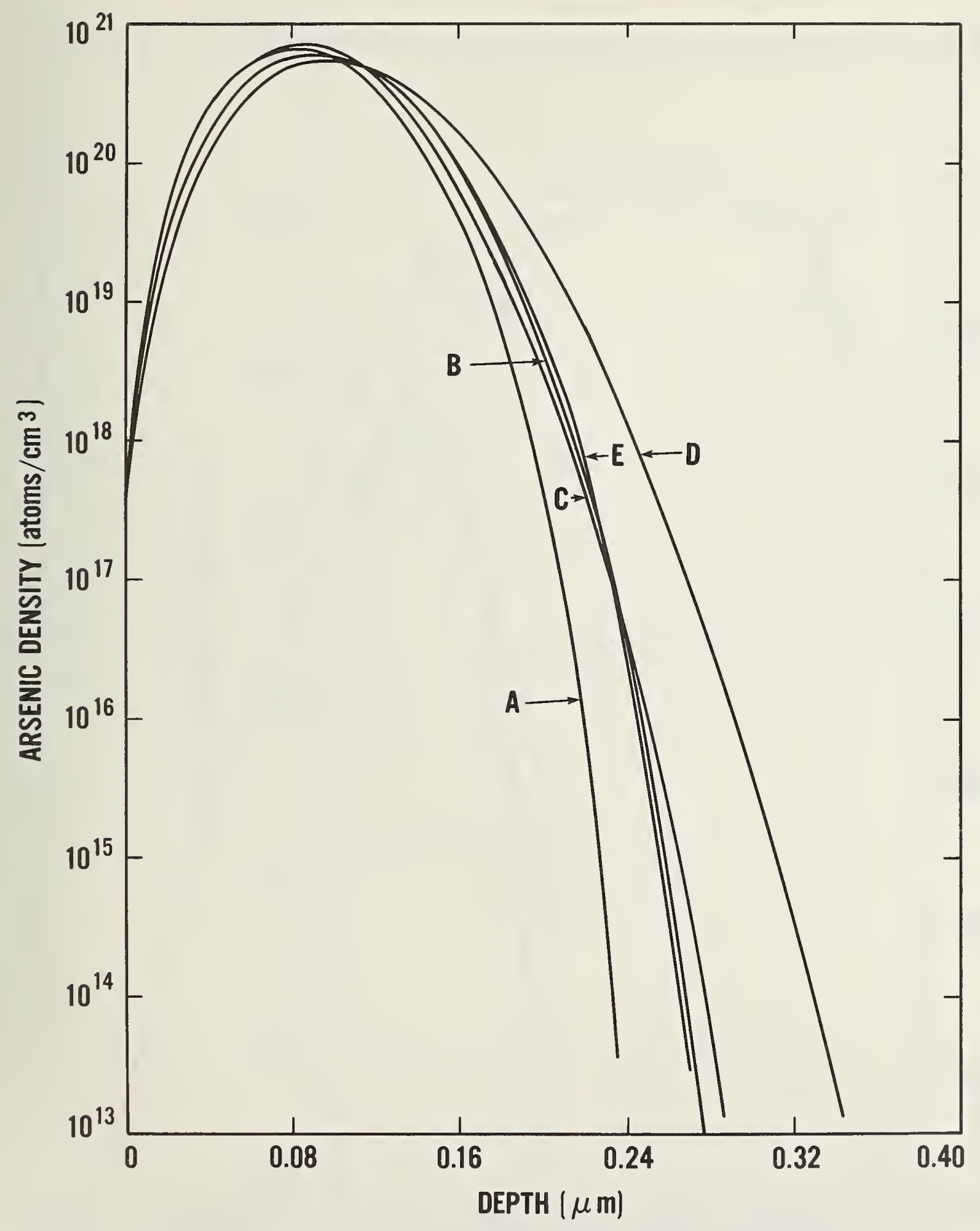

Figure 3.2 Predictions of the winterbon amoxphous target code with several choices of the potential for the case of a 150-kev implant of arsenic into silicon at a dose of $5 \times 10^{15} \mathrm{~cm}^{-2}$. The potentials used were: Thomas-Fermi (Lindhard) (A), Chu-Murley (Wilson) (B), Moliere (C), Lenz-Jensen (D), and the Kalbitzer (Wilson) (E) forms. It can clearly be seen that the potentials predict approximately the same peak location but rathex different junction depths. 


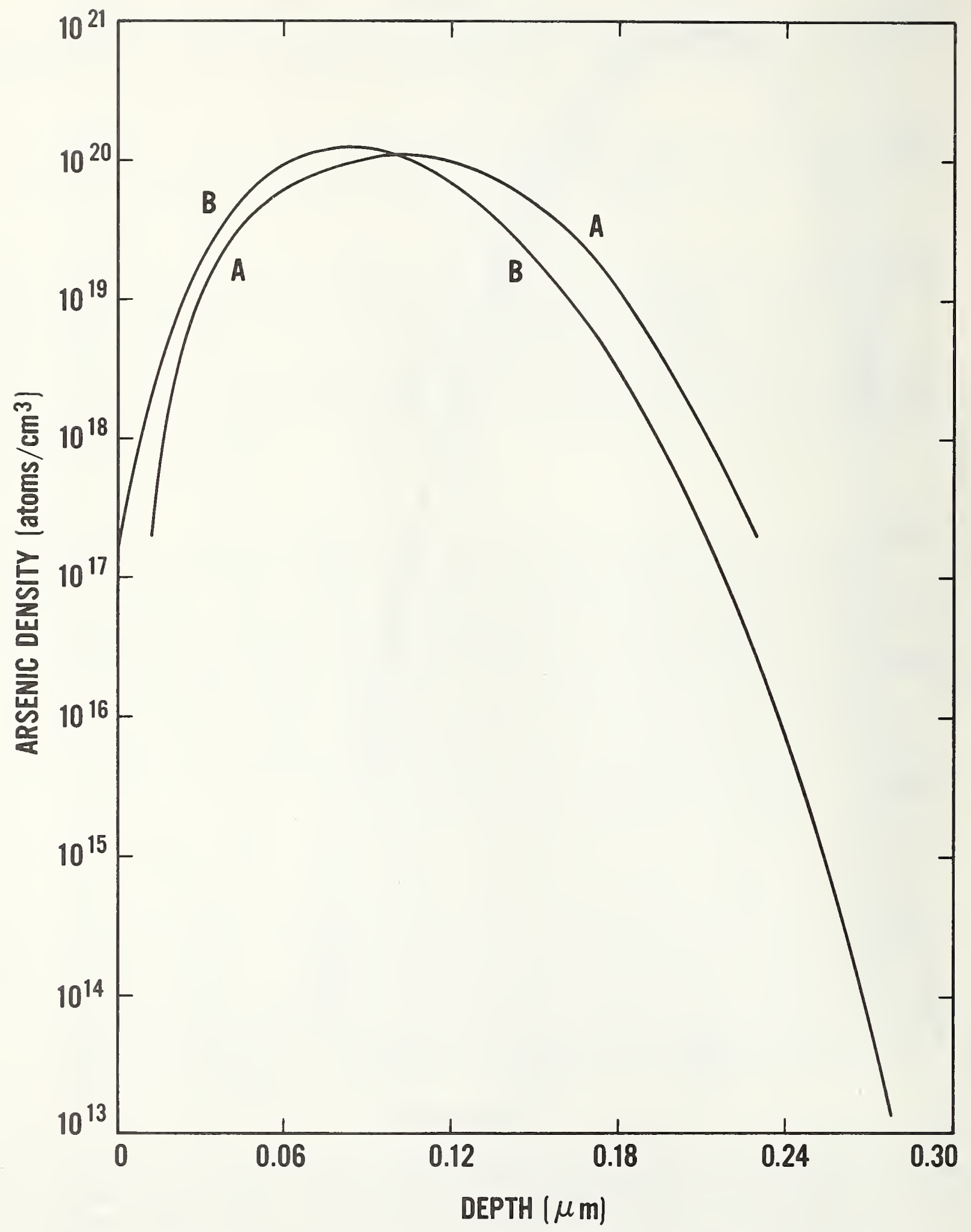

Figure 3.3 Comparison of the TRIM (Monte-Carlo) code (A) and the Winterbon amorphous target code (B) for $150-\mathrm{keV}$ implant of arsenic into silicon at a dose of $1 \times 10^{15} \mathrm{~cm}^{-2}$. Both calculations make use of the Moliere potential. 


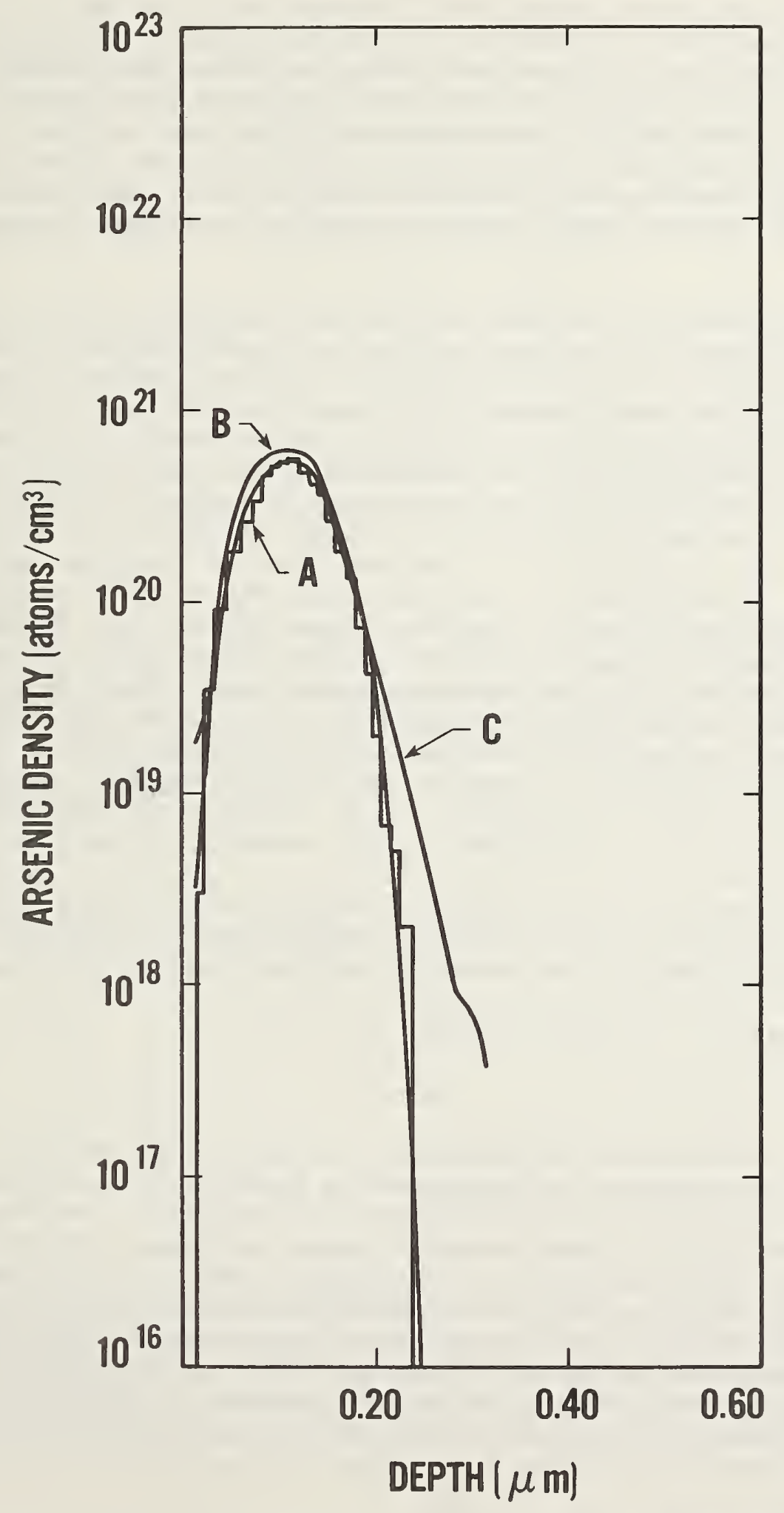

Figure 3.4 Comparison of profiles for $150-\mathrm{keV}$ arsenic implanted into silicon. Curve A: TRIM code (Moliere); curve B: analytic code (WHB average potential); curve $C$ : atomic profile as determined by secondary ion mass spectroscopy. 
tions tend to decrease more rapidly with distance away from the surface than do the SIMS. A "tail" in the distribution has been seen by other techniques and has been largely attributed to ion channeling during the initial stages of the implant (before a continuous amorphous layer had been formed) [17]. The topic of ion channeling will be discussed in the next section. Nevertheless, from figure 3.4 it is seen that the amorphous target codes provide an excellent estimate of ion ranges for the majority of ions implanted into the crystal under "random equivalent" (minimum channeling) conditions.

From the point of view of the prediction of one-dimensional ion ranges in amorphous targets, each of the above approaches has its strong and weak points. The winterbon code is relatively fast, but relies upon a seemingly inconsistent method for the construction of the distribution from the calculated moments. Nevertheless, the fact that a finite number of parameters can be found to describe a continuous distribution allows rapid estimation of trends and thus a useful scaling for prediction of implant distributions. The Monte-Carlo code, on the other hand, follows the details of the motion of the incident ion and constructs the histogram from the histories of the incident particles. In the region of the peak, where there are a significant number of the incident particles which have come to rest, the effects of noise are minimized. However, in the tail of the distribution where the concentration is approaching the substrate dopant level (forming a junction if the substrate is of the opposite conductivity type), there is usually an insufficient number of incident particles which have come to rest so as to be able to predict such important parameters as junction depths. Basically, the noise level of the data in that region, being inversely proportional to the square root of the number of particles in the interval, is very large.

Hence, both techniques have their limitations for calculating one-dimensional distributions. Nonetheless, the agreement between the two predictions for depth distribution of dopants as presented in the figure is very good.

\section{C) Ion Range Theories for Point Sources}

\section{i) Introduction}

As has been discussed in the introduction, two-dimensional effects dominate the operating characteristics of fine-geometry semiconductor devices. For this reason, the prediction of the implanted ion density in two dimensions and its evolution during thermal annealing are of critical importance. Fortunately, Monte-Carlo techniques are particularly well suited to the calculation of ion range distributions in two dimensions, while analytic theories suffer from difficulties in reconstructing multidimensional distributions from their moments. As all of the physics of the ion penetration process are identical for one-dimensional and for multidimensional range calculations, the only differences in the codes are the extraction of additional parameters.

\section{ii) Analytic Theories}

The question of lateral spreading of the ion beam was initially addressed by Lindhard et al. [29], who developed an integro-differential equation for the lateral spreading of an ion heam along with the integro-differential equation 
for the depth distribution of implanted ions. Winterbon et $a$. [36] were able to solve these equations for a power law potential to obtain estimated equal density contours to 10 percent of peak density by summing a total of 30 moments in the incident and transverse dimensions. Since such a calculation would require major revisions of the Winterbon range code for each ion-energy combination chosen, this method was not adopted in the present study. The other approaches to constructing two-dimensional distributions from analytic solutions require the use of simplifying assumptions.

Furukawa et al. [41] assumed a Gaussian distribution in three dimensions as the shape of the ion distribution from a point source. This particular mathematical form of a Gaussian has particular advantages in constructing the distributions that result from scattering of a distribution under a vertical mask edge. The difficulty with applying the Gaussian approximation is that range distributions, even in one dimension, are rarely Gaussian - especially for common implants in silicon such as boron [42] or arsenic (e.g•, [43]).

\section{iii) Monte-Carlo Codes}

While difficulties arise in estimating lateral contours from analytic codes, the application of Monte-Carlo codes to lateral penetration problems is quite straightforward, since the code must follow the three-dimensional path of the ion to calculate the total energy loss along its trajectory. Thus, the program need only note the resting point in three dimensions instead of just the vertical component of the rest position.

As before, the major difficulty in applying Monte-Carlo techniques is that a significant number of histories must be followed to obtain reasonable statistical accuracy. However, since the ion rest position is being noted in two dimensions, more histories (typically at least 10,000) are required. In two dimensions, the Monte-Carlo techniques share a common disadvantage with the analytic techniques in that there is no convenient (but accurate) finiteparameter description of two-dimensional density contours. A Monte-Carlo approach to the spreading of boron implanted into silicon was obtained by Shimizu et al. [44], who found good agreement not only with the depth profile of dopants but also with junction staining experiments as well. They also found that in addition to not being Gaussian in the vertical direction, neither was the lateral spreading well described by a Gaussian.

In the present study a slightly modified version of the TRIM code [37] was used to model the lateral spreading process. The TRIM code has incorporated techniques that reduce computer usage by an order of magnitude for the same number of histories without sacrificing numerical accuracy, thus making the calculation of the increased number of trajectories needed to calculate twodimensional distributions feasible. The code was modified to quantize the lateral spreading in cylindrical coordinates (for point source distribution) or in rectangular coordinates (for calculation of scattering under a mask edge).

\section{iv) Comparison of Predictions}

Shown in figure 3.5 are the equal density contours in cylindrical coordinates for $75-\mathrm{keV}$ arsenic implanted into amorphous silicon at 0-deg tilt. The pre- 

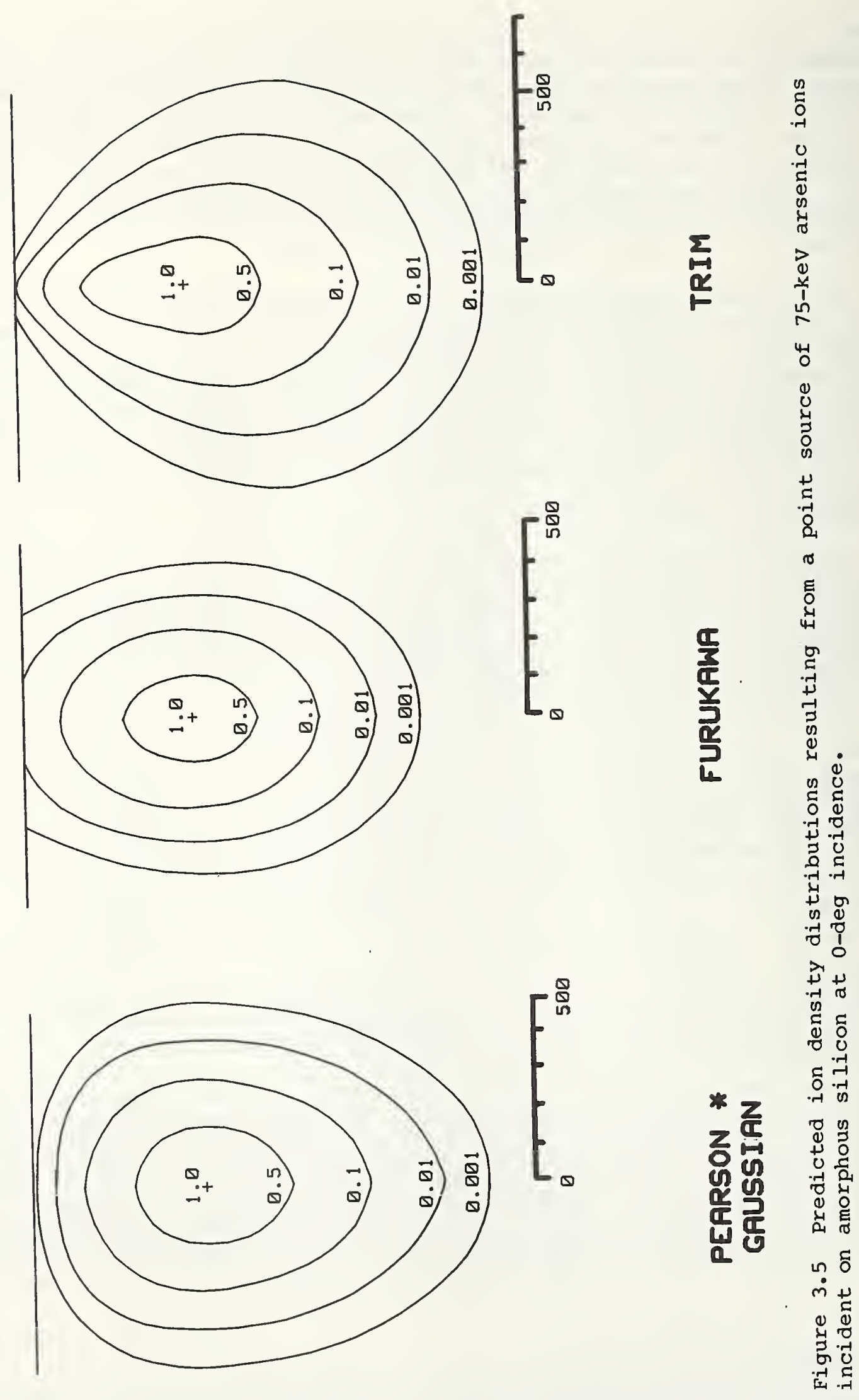
dictions of the Furukawa theory, the Winterbon code using a Pearson distribution vertically multiplied by a Gaussian distribution laterally (following [45]), and the TRIM code are shown. From this figure it can be seen that the lateral spreading, as well as the spreading in the vertical direction, as calculated by the TRIM code, varies drastically from that which would be expected on the basis of a Gaussian distribution, with the maximum lateral penetration occurring at the projected range $(\sim 50 \mathrm{~nm})$ for the assumption of uniform lateral scattering, while the TRIM code predicts maximum lateral penetration at roughly three times the projected range ( 150 nm).

D) Scattering Under a Rectangular Mask Edge

\section{i) Introduction}

When the ion density distribution in a solid $F(x, y, z)$ resulting from a point ion source is known (as was solved in the last section), the density distribution $G(x, y, z)$ resulting from implantation into an unmasked surface $A(y, z)$ follows from superposition:

$$
G(x, y, z)=\int_{A} F(x, y-\xi, z-\eta) d \xi d \eta .
$$

Thus, as was previously mentioned, the accuracy in calculating the ion scattering under a mask edge is directly related to the accuracy for which the point source ion distribution can be obtained.

\section{Comparison of Theories}

The advantage in assuming a Gaussian lateral distribution for a point source is that the solution for implantation into a rectangular mask edge can be expressed in closed form as a complementary error function [41]. The alternative approach, adopted by Ryssel et al. [45] is to multiply the vertical distribution by a Gaussian (for reasons that are not readily apparent). When Monte-Carlo simulation techniques are applied to a grid with depth resolution element $\Delta$, eq $(3.14)$ becomes

$$
G(x, y, z)=\sum_{n, m} F(x, y-n \Delta, z-m \Delta),
$$

where the indices $\mathrm{n}$ and $\mathrm{m}$ are chosen to fill the area $\mathrm{A}$.

The results of these calculations are shown in figure 3.6 for $75-\mathrm{keV}$ arsenic implanted into silicon at 0 -deg incidence. From this figure, it is seen that the distribution as predicted by the Monte-Carlo codes (without the use of simplifying or ad hoc assumptions) penetrates laterally approximately half the vertical penetration and deviates markedly from the analytic solutions, especially in the depth of the maximum lateral penetration. 


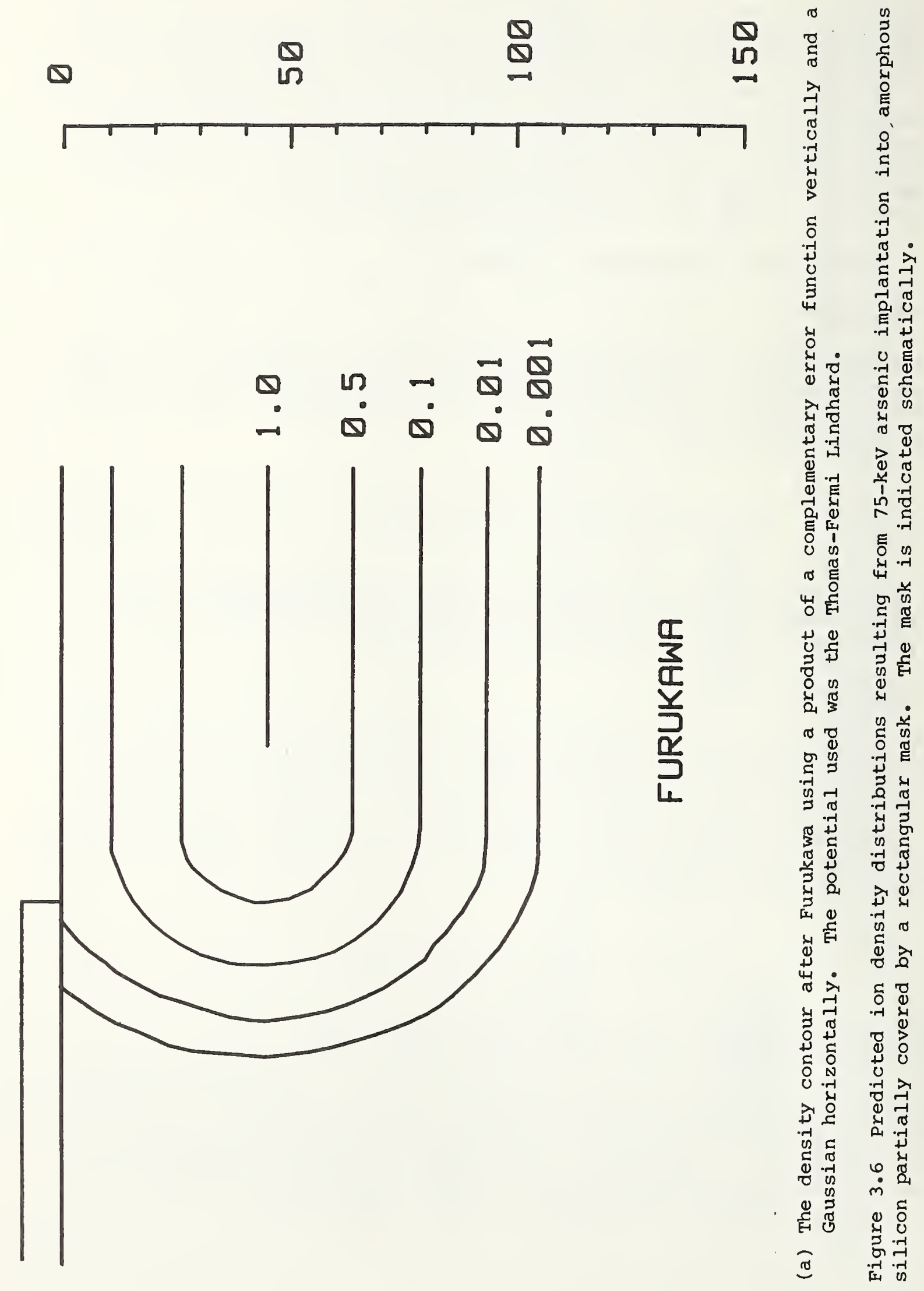




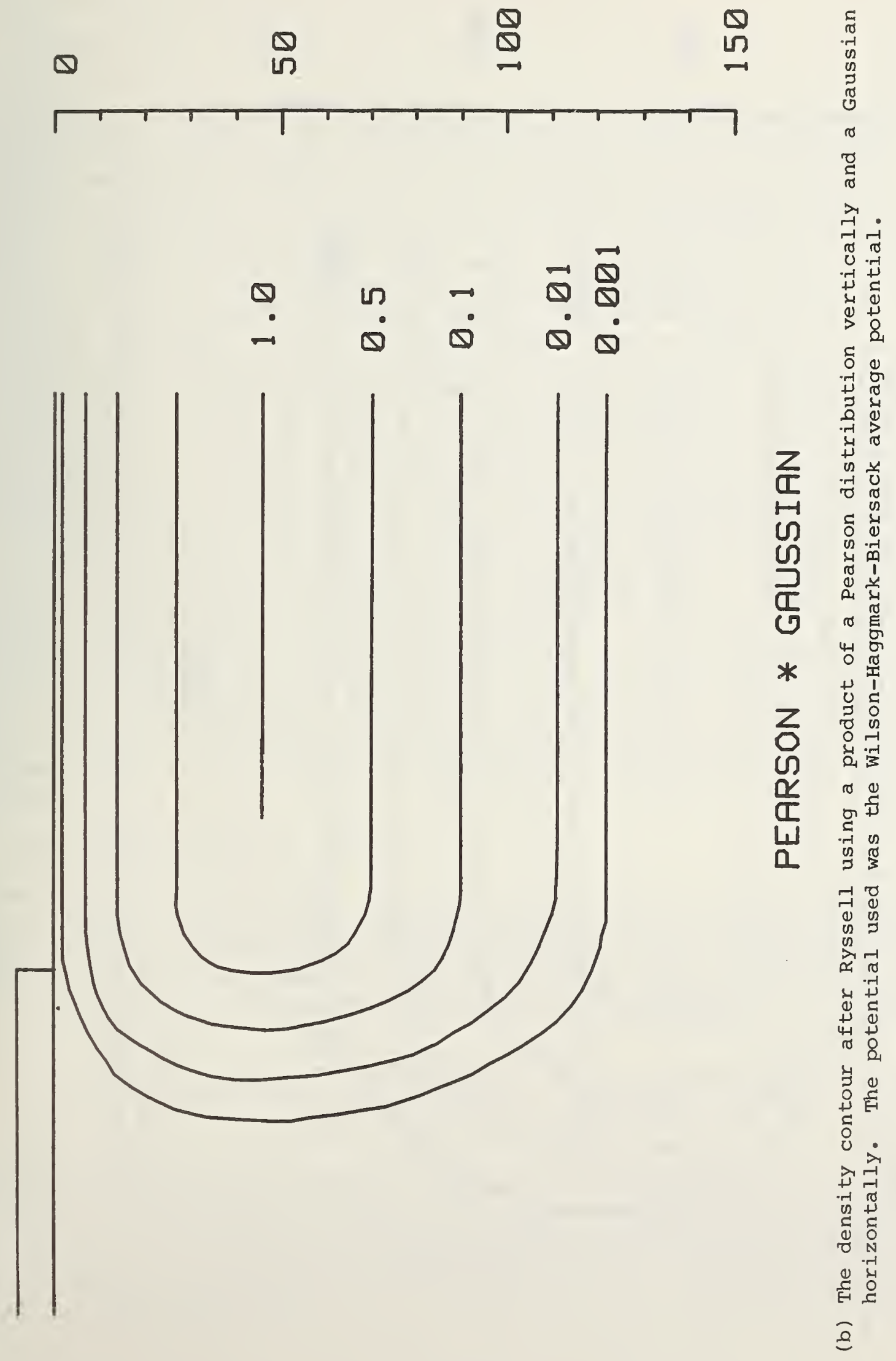



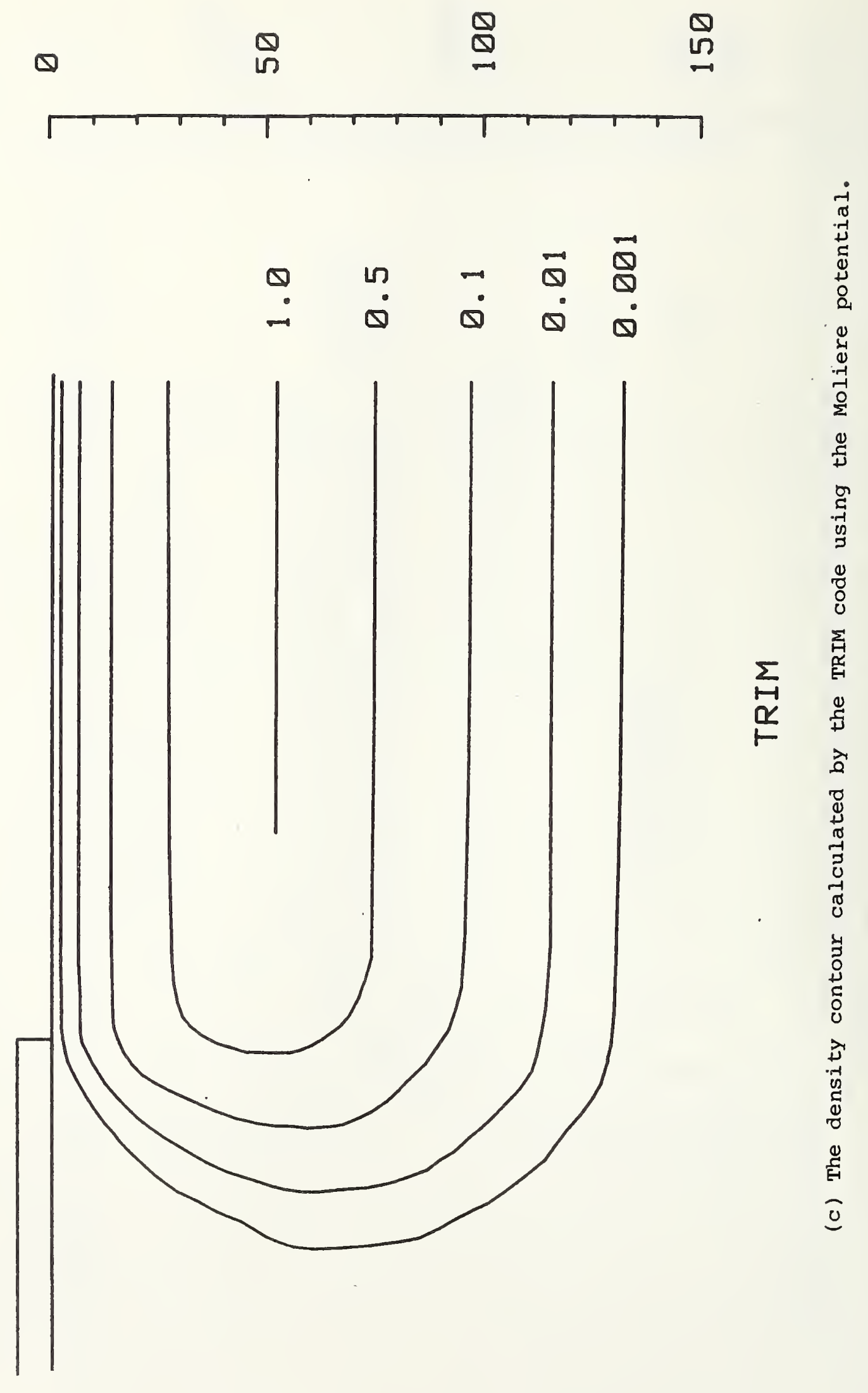


\section{E) Ion Channeling}

\section{i) Introduction}

The discussion of ion ranges has thus far been concerned with implantation into amorphous targets. When implantations are performed into crystalline targets, there is the possibility that the incident ions will travel much farther than would be predicted by range theories for amorphous targets. This excess penetration is caused by the correlated small-angle collisions between the incident ion and substrate atoms lying along low-index crystallographic directions which prevent the transfer of large amounts of the energy of the incident ion to substrate atoms (i.e., the trajectories reduce or eliminate nuclear stopping effects). Thus, ions that follow that type of trajectory encounter a greatly reduced stopping power and thus penetrate significantly greater distances compared to those ions which follow a trajectory for which the substrate atoms appear randomly arranged. The topic of ion channeling has been the subject of a number of excellent reviews (e.g., $[46,47])$, and has been understood both on the basis of Monte-Carlo simulations and also as a result of analytic theories. This section employs the analytic model of Lindhard [18] since this model is of sufficient generality to provide a means to characterize a wide variety of implantation parameters and to explain trends in the data. Lindhard's model begins with the concept of a string of atoms, which leads to the classification of an ion's trajectory in a single crystal according to its "transverse energy," i.e., the component of ion's energy in the plane perpendicular to the atomic string. Classification of the ion's trajectory according to transverse energy in turn leads to the concept of a critical angle. It is then shown that while the critical angle describes only the limit of applicability of the continuum model, this concept provides a useful means of characterizing the implantation conditions. The effect of amorphous surface layers will be discussed and the effects of ion channeling on the lateral implant profile will then be discussed.

\section{ii) The Continuum Model of Lindhard}

To model the channeling effect, Lindhard approximated the substrate lattice by a series of strings of atoms parallel to the low index crystalline directions. If $V(r)$ is the potential energy between an isolated atom and the incident ion, then the potential of an ion due to an infinite string of atoms at an average spacing $d$ along the $x$-axis ( $f i g .3 .7$ ) is given by:

$$
V_{T}=\sum_{n=-\infty}^{\infty} v\left(r_{n}\right)=\sum_{n=-\infty}^{\infty} v\left(\sqrt{\rho^{2}+z^{2}}\right)=\sum_{n=-\infty}^{\infty} v\left(\sqrt{p^{2}+n^{2} d^{2}}\right) .
$$

Lindhard then argued that since the ions are moving with high velocity, and since the collision anqles are small for channeled trajectories, the channeled ion would interact with many atoms at once. For these ions, details of the atomic nature of the lattice would become blurred, so that the atomic distribution would appear uniform. Mathematically, this would imply the limit of a continuous string 


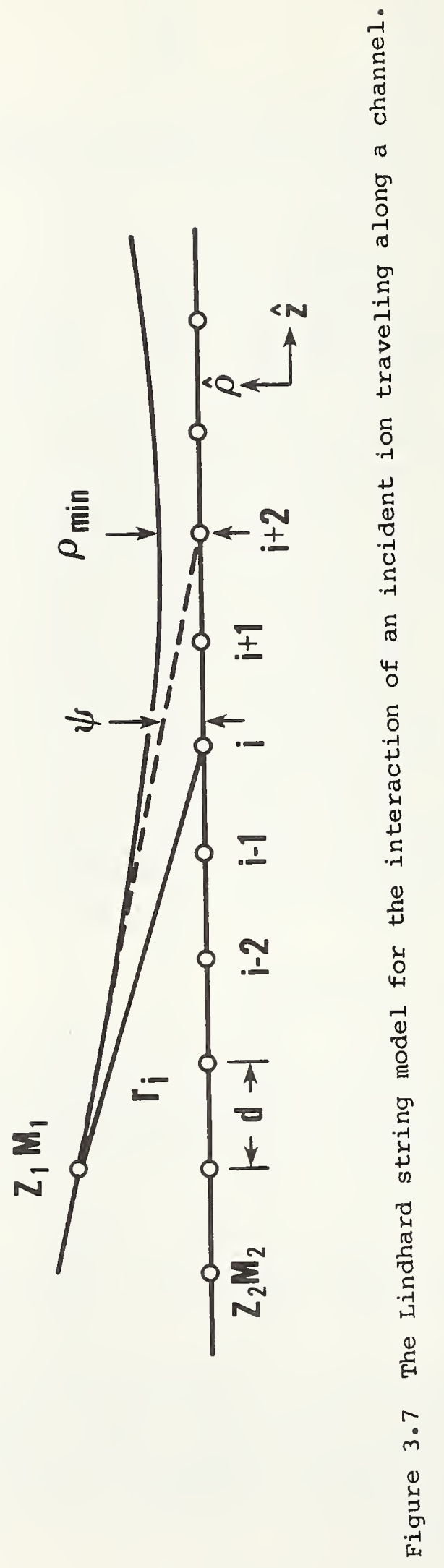




$$
V_{T}+\frac{1}{D} \int_{-\infty}^{\infty} V\left(\rho^{2}+z^{2}\right)^{1 / 2} d z \equiv U(\rho) .
$$

Since the potential energy now depends only on $\rho$ (the distance from the string), the only effective forces on the ion are normal to the row. This treatment neglects the contribution of electronic stopping which, since it is purely frictional, provides no directional effects. The model has thus reduced the classification of the ion's trajectory in a three-dimensional crystal to the problem of the motion of the ion in a plane perpendicular to the atomic string. Neglecting electronic stopping, the total energy of the ion in this transverse plane $\left(E_{\perp}\right)$ is a constant, equal to the sum of the potential energy $U(\rho)$ plus the transverse kinetic energy $E_{K \perp}$. For an ion incident to the string at an angle $\psi$ with initial kinetic energy $E$ given by

$$
E=\frac{1}{2} M_{1} v^{2} \text {, }
$$

where $M_{1}$ is the mass of the ion and $v$ is the incident velocity of the ion, the transverse kinetic energy is given by

$$
E_{K \perp}=\frac{1}{2} M_{1}(v \sin \psi)^{2} \approx \frac{1}{2} M_{1} v^{2} \psi^{2}=E \psi^{2} \text {. }
$$

Thus, the total transverse energy is the sum of the kinetic energy plus the (repulsive) potential energy of the ion with respect to the string

$$
E_{\perp}=U(\rho)+E_{K \perp}=U(\rho)+E \psi^{2}
$$

According to the continuum model, ion trajectories can be classified according to their transverse energy, the effects of which on ion range profiles has been discussed in [19]. Of greater concern in the present study are the conditions under which an ion can be expected not to channel. Ions with transverse energy greater than some critical value $\left(E_{C}\right)$ approach the string of atoms closely enough to see the substrate atoms as individual scattering centers. Transverse energy is no longer conserved, and the continuum model of the atomic string no longer holds. Since the orderly arrangement of lattice atoms plays no part in influencing the trajectory of this last class of ions, the substrate atoms are randomly arranged in regard to the ion's motion. The distribution of this type of ion should therefore be described by the range theories for amorphous targets.

The continuum model does not predict channeling ions with transverse energy above the critical value $E_{C}$. By eq $(3.20)$, the transverse energy is a sum of a potential energy term and a kinetic energy term. Two concepts are related to that of a critical transverse energy, i.e., the critical approach distance and the critical angle. The critical approach distance $\left(\rho_{\mathrm{C}}\right)$ is the distance from an atomic string within which an ion can approach the string with zero transverse kinetic energy $(\psi=0)$ and can still exceed the crjitical energy. The critical angle $\left(\psi_{C}\right)$ is the angle at which an ion can enter the crystal in a region of zero potential energy $(U=0)$ and have transverse kinetic energy equal to the critical energy. Thus,

$$
E_{C}=E \psi_{C}^{2}=U\left(\rho_{C}\right)
$$


The ability to predict channeling behavior from the continuum model thus reduces to the problem of finding the critical approach distance $\left(\rho_{C}\right)$ as a function of incident ion energy E. Fortunately, a solution to this problem has been given by Morgan and Van Vliet [20], on the basis of computer modeling. Using their model to calculate the minimum approach distance ( $\rho_{\text {min }}$ ) and including the thermal vibrations of the lattice, urms (typically as modeled in the Debye approximation [47]), the critical approach distance is given by [46]:

$$
\rho_{c}^{2}=\rho_{\text {min }}^{2}+u_{r m s}^{2} .
$$

The critical angle can then be calculated from eq (3.21) by substituting $\rho_{\mathrm{C}}$ into the expression for string potential,

$$
\psi_{C}=\sqrt{U\left(\rho_{C}\right) / E} \text {. }
$$

Parameters for the implant conditions studied here are summarized in table 3.2.

It is important to realize that the calculated critical angle corresponds only to the limits of applicability of the continuum model and not to the angular extent of channeling. A number of studies $(e . g \bullet,[22,46])$ have demonstrated that channeling characteristics typically continue until the ion beam is oriented at least twice the classical critical angle from the nearest low index direction. Nevertheless, the critical angle does provide a convenient scaling parameter to characterize implant conditions and can be used to calculate the substrate tilt angles for which unintentional channeling can be minimized [22]. In addition, the continuum model can also be used to estimate the effects of amorphous surface layers on trajectories in underlying crystals and to estimate the effect of channeling on lateral profiles.

\section{iii) The Effect of Amorphous Surface Layers}

Implantations into silicon for the fabrication of electronic devices are often performed through amorphous surface layers resulting from surface oxidation or from ion sputtering [48]. The effect of a surface amorphous layer is to introduce an angular divergence in the ion beam due to nuclear collisions in the amorphous layer [49]. The result of this angular divergence is a distribution of angles at which the ions transmitted through the amorphous surface layer encounter the crystallographic axes of the substrate, and thus a variation in the probability of ion channeling ( $i . \epsilon_{.}$, deep penetration) by the incident ions [23].

This decrease in the probability of ion channeling can be estimated using a procedure developed by Lugujjo and Mayer [50]. This method approximates the variation in the probability of ion channeling from nearly unity at direct angular alignment to zero probability at twice the critical angle by a step function probability at the critical angle; i.e., in this approximation, an ion has unity probability for channeling when incident at an angle above the critical angle (fig. 3.8). This assumption is essentially equivalent to assuming that the probability of ion channeling as a function of angle of incidence is symmetric about the critical angle when integrated over an angular distribution of finite width. Note that this is not equivalent to claiming an ion implanted directly into a crystalline substrate would have zero 
TABLE 3.2 - Dechanneling of Arsenic Implants into (100) Silicon by Amorphous Surface Layers for 0-Deg Incident Beam.

$\begin{array}{lrccc}\begin{array}{c}\text { Amorphous } \\ \text { Layer }\end{array} & \begin{array}{r}\text { Energy } \\ (\mathrm{keV})\end{array} & \begin{array}{c}\rho_{\mathrm{C}} \text { in } \\ \text { substrate } \\ (\mathrm{nm})\end{array} & \begin{array}{c}\theta_{\mathrm{C}} \text { in } \\ \text { substrate } \\ \text { (degrees) }\end{array} & \begin{array}{c}\text { Thickness for } \\ >90 \% \text { dechanneling } \\ \text { (nm) }\end{array} \\ \mathrm{Si} & 75 & 0.0349 & 3.93 & 8.04 \\ \mathrm{Si} & 150 & 0.0274 & 3.72 & 14.0 \\ \mathrm{SiO}_{2} & 75 & 0.0349 & 3.93 & 7.41 \\ \mathrm{SiO}_{2} & 150 & 0.0274 & 3.72 & 12.9\end{array}$




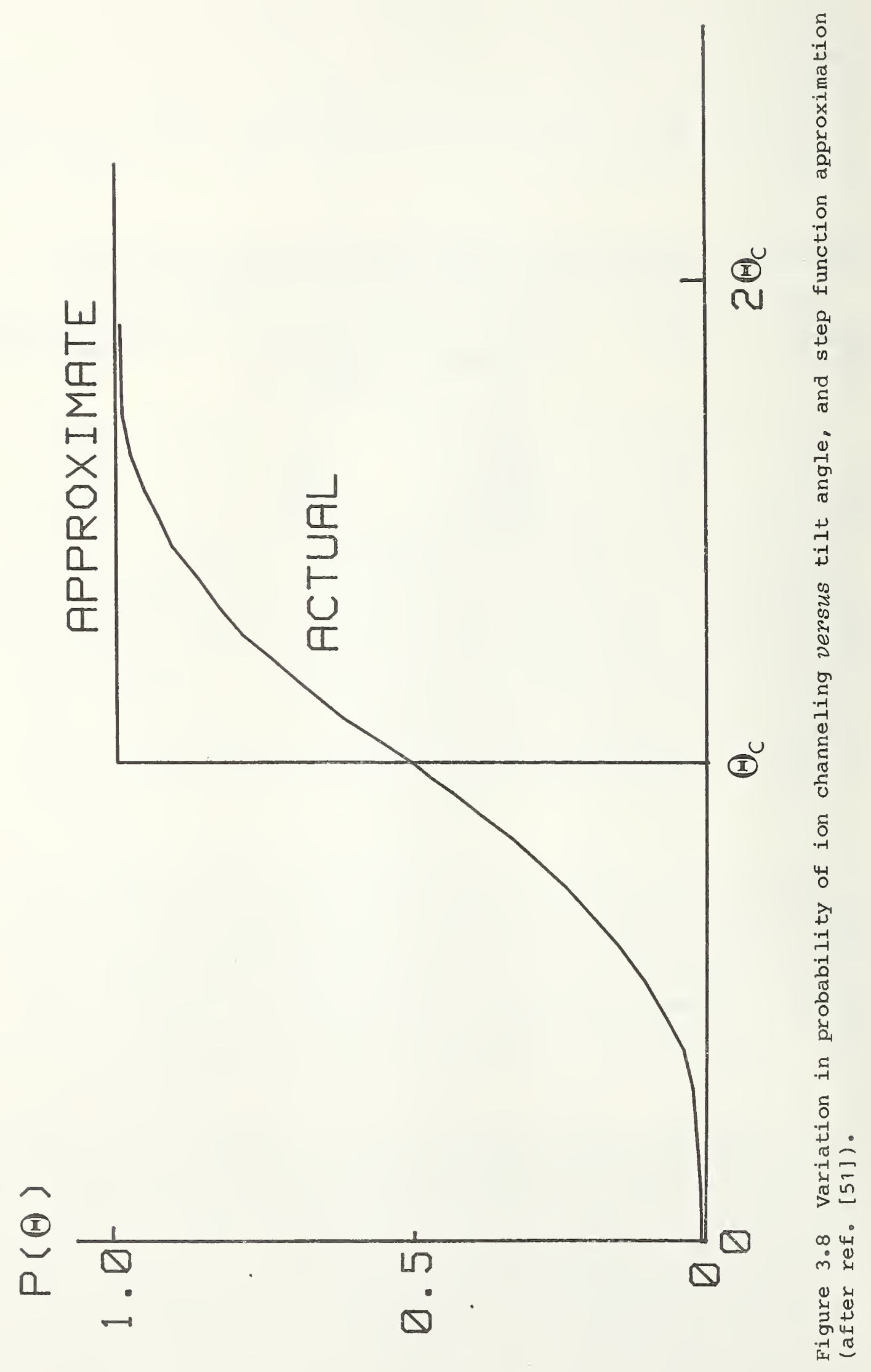


probability of channeling for alignment at angles greater than the critical angle. The actual angular spreading particular to each set of implant conditions is estimated from the plural scattering theory of Meyer [49]. The amorphous layer thicknesses required to produce 90-percent dechanneling of the ion beam for the arsenic implant conditions studied here are shown in table 3.2, where it is seen that only $14 \mathrm{~nm}$ of surface damage or surface oxide would be required to totally dechannel the incident ion beam.

\section{iv) "Lateral Channeling"}

Ion channeling has been shown to strongly affect the depth distribution of dopants resulting from implantation into initially crystalline targets. A natural extension is to inquire to what extent implantation will modify the lateral distribution of dopants. In a (100)-oriented wafer, both the [010] and [001] directions run perpendicular to the [100] surface normal and are atomically identical. By employing the physical principles described in preceding sections, however, it is possible to show that there is negligible probability of ions channeling parallel to the implanted surface.

Earlier, it was pointed out that ions cannot channel parallel to an atomic string if they start their trajectories at too high an angle to the string or if their trajectories begin too near an atomic string. According to table 3.2 , the closest an ion can get to the string for these implants in the continuum model and still channel is approximately $3 \times 10^{-9} \mathrm{~cm}$. Collisions that produce large-angle scattering require impact parameters of approximately $10^{-12} \mathrm{~cm}$ [51]. Thus, collisions that will deflect the incident ions through an angle large enough to encounter a low index lateral direction also require the ion to start out with too large a potential energy transverse to the lateral channel. For this reason, channeling in the lateral direction is a highly unlikely process, and calculations of lateral penetration from the amorphous target codes should accurately predict the contours for the implants into crystalline substrates as well.

\section{F) Electrical Activation of Arsenic-Implanted Silicon}

Previous discussions have described the methods used to predict the resting points of the implanted ions. However, to fabricate electronic devices, these implanted impurities must be made electrically active by incorporation into the silicon lattice. The electrical activation of arsenic implanted into silicon has been studied for laterally uniform implants, and the understanding thus obtained will he briefly reviewed here.

Room temperature implantation of arsenic at doses above $3 \times 10^{14} \mathrm{~cm}^{-2}$ leads to the formation of an amorphous layer at the silicon surface [52]. This layer regrows epitaxially at temperatures below $600^{\circ} \mathrm{C}$, with no detectable redistribution. This regrowth produces excellent incorporation of the implanted arsenic on lattice sites and complete electrical activation of these impurities up to a solubility limit of approximately $3 \times 10^{20} \mathrm{~cm}^{-3}$ [53]. For (100)-oriented silicon substrates (as are examined in this study), no lattice disorder has been detected by $\mathrm{MeV}$ ion channeling techniques [53]. These studies also determined that incomplete electrical activation of the implant arsenic (for (100) silicon) occurred only near the peak of the ion distribution, where the implanted ion density exceeded the solubility limit. Thus, 
the $600^{\circ} \mathrm{C}$ annealing temperature exanined in this study should provide an electrically active marker of the implanted arsenic distribution for arsenic densities helow the solubility limit. The other annealing temperatures examined in this study $\left(1000^{\circ} \mathrm{C}\right)$, and the fluence level chosen, correspond to arsenic densities that will redistribute by concentration dependent diffusion effects [53] - the understanding of which is important to device process modeling. 


\section{EXPER IMENTAL}

In this section the experimental techniques used in this study are reviewed, and the results obtained by each are presented. First, the details of the sample preparation are presented. The spreading resistance technique and results, the etch technique and results, and the induced current technique and results are then discussed in turn.

\section{A) Sample Fabrication}

Initial sample preparation was performed at Hughes Research Laboratories. A 50-nm oxide was grown on boron-doped silicon wafers. The oxide was then coated sequentially with $1.2 \mu \mathrm{m}$ of photoresist, $0.2 \mu \mathrm{m}$ of aluminum, and $1 \mu \mathrm{m}$ of photoresist. The top layer of resist was exposed in a grating pattern (alternating lines and spaces) of either $6.7-\mu \mathrm{m}$ or $13-\mu \mathrm{m}$ period. This layer was developed and etched using conventional wet chemistry. The aluminum was then plasma etched. The bottom layer of resist and the resist over the aluminum was then reactive ion etched. Finally, the oxide was etched in a conventional acid etch. The resulting mask structure is shown schematically in figure 4.1. The reactive ion etch was used to create extremely vertical walls in the resist. The success of this technique can be seen in fiqures 4.2 and 4.3, which show typical patterned wafers. These vertical walls assure that none of the implanted ions would penetrate through the mask near the edge of the junction.

The wafers were then implanted using the goniometer end station at Hughes [19]. All the wafers were (100) oriented, and the grating pattern was also lined up in the $(100)$ direction. All the implants were $5 \times 10^{15}$ arsenic ions $/ \mathrm{cm}^{2}$. The samples were tilted with respect to the ion beam, parallel to the grating lines, at four angles: 0, 4, 7 and $10 \mathrm{deg}$. Two implant energies were used: $75 \mathrm{keV}$ and $150 \mathrm{keV}$.

At NBS the photoresist and aluminum were stripped off in acetone and the wafers were cleaned using a standard peroxide solution [54]. The wafers were then annealed in dry nitrogen. Two annealing temperatures were used: $600^{\circ} \mathrm{C}$, to remove the initial damage and activate the As with minimal redistribution and $1000^{\circ} \mathrm{C}$, to determine the effect of diffusion on ultimate junction shape. The wafers were then thinned from the back side. This step was found necessary to minimize crack and step formation on the cleaved surface. Finally, the wafers were cleaved perpendicular to the grating fingers.

Two final steps were employed. For the etch technique, the cleaved edge of the sample was etched in a solution of two-percent hydrofluoric acid in nitric acid in ambient light for $5 \mathrm{~s}$. This photosensitive etch selectively attacks n-type, but not $p$-type, silicon. Care was taken to try to contact only the cleaved edge with the etch. The samples were then rinsed in water. For the induced-current technique, a contact was deposited on the top surface. Typically, silver paint was used and contact was therefore made in parallel to a large number of junction stripes.

Following the photochemical etch, the samples were mounted in the scanning electron microscope for examination using conventional secondary electron imaging techniques. It was not necessary to coat the samples. We did find 


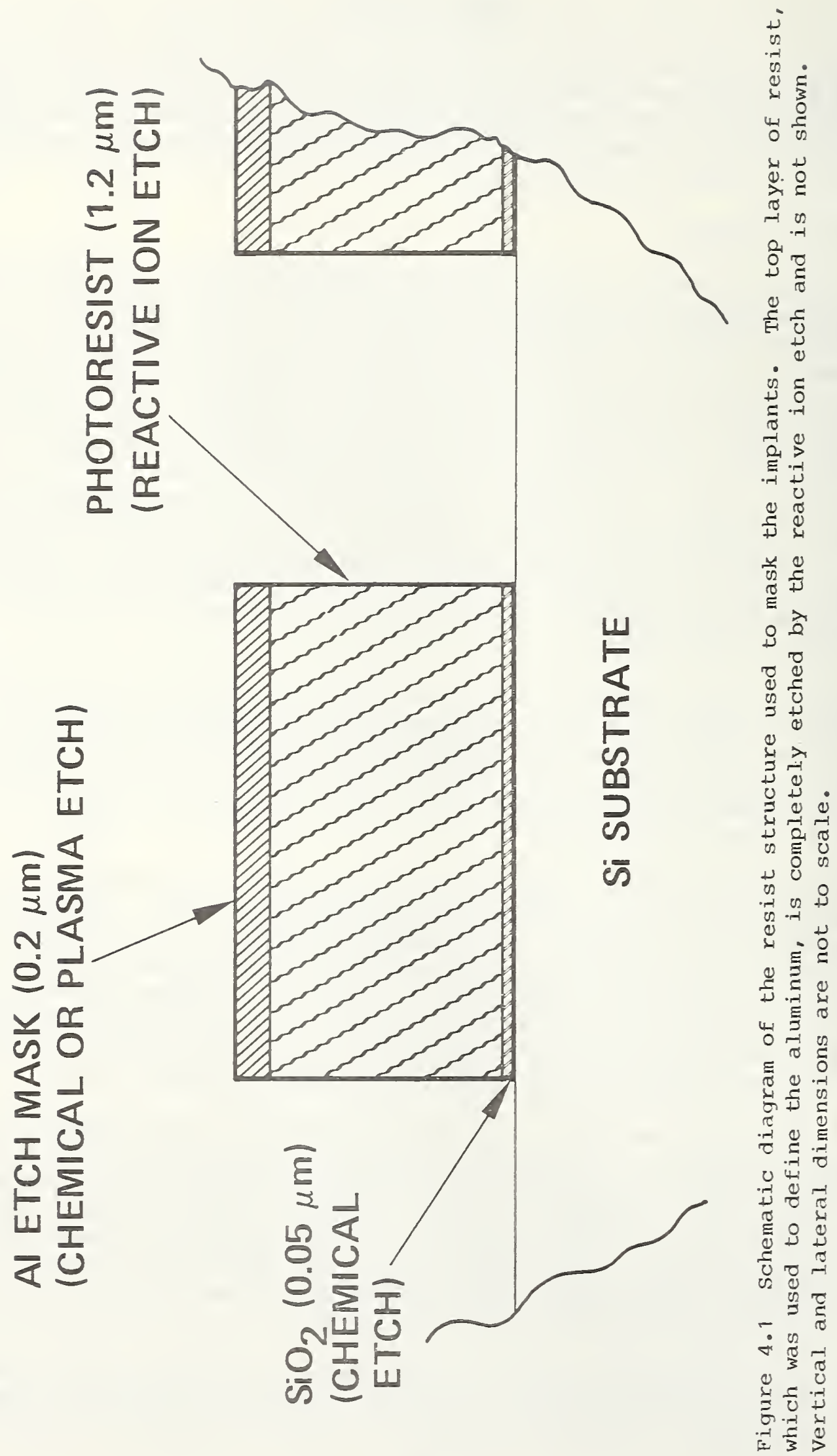




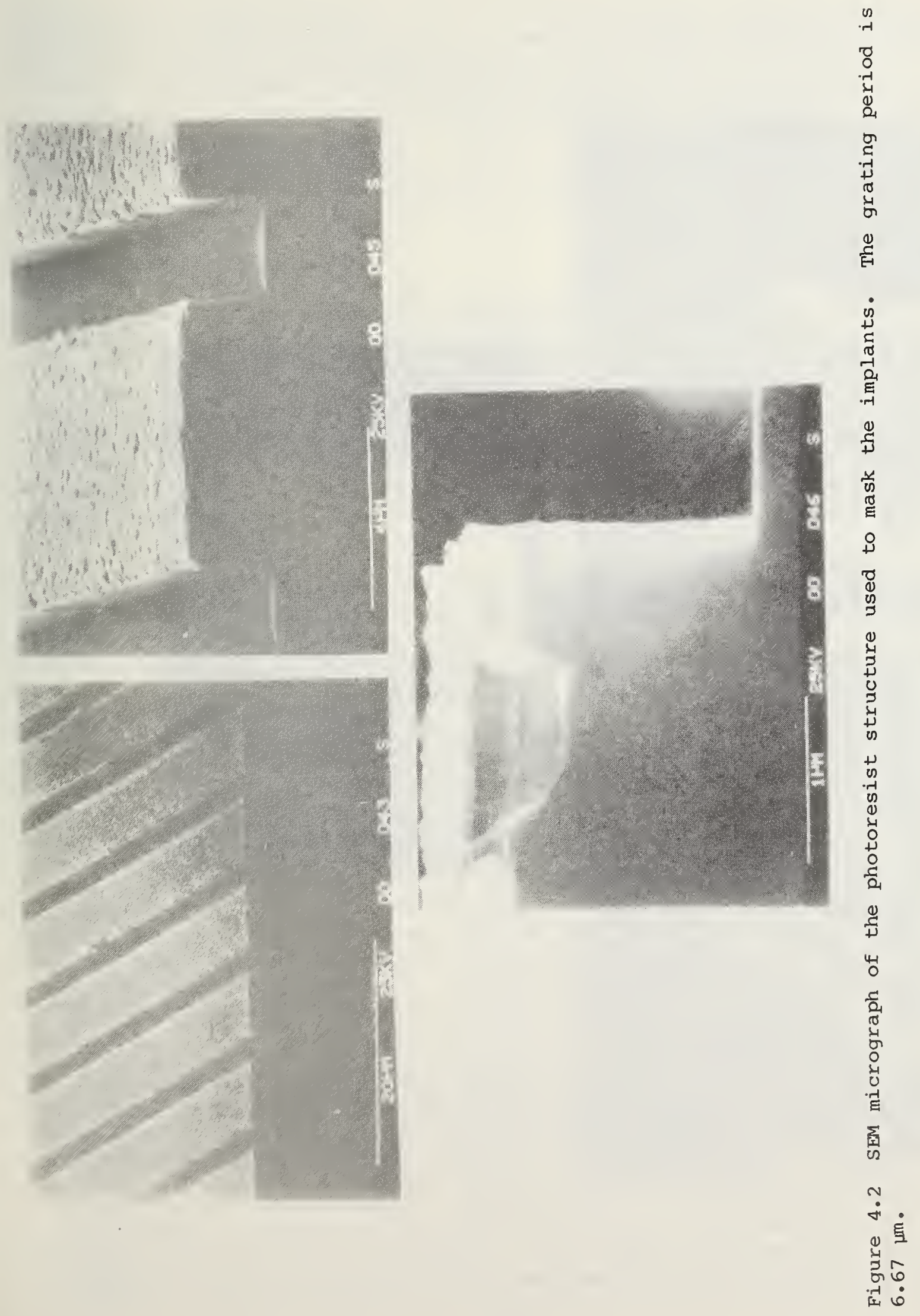




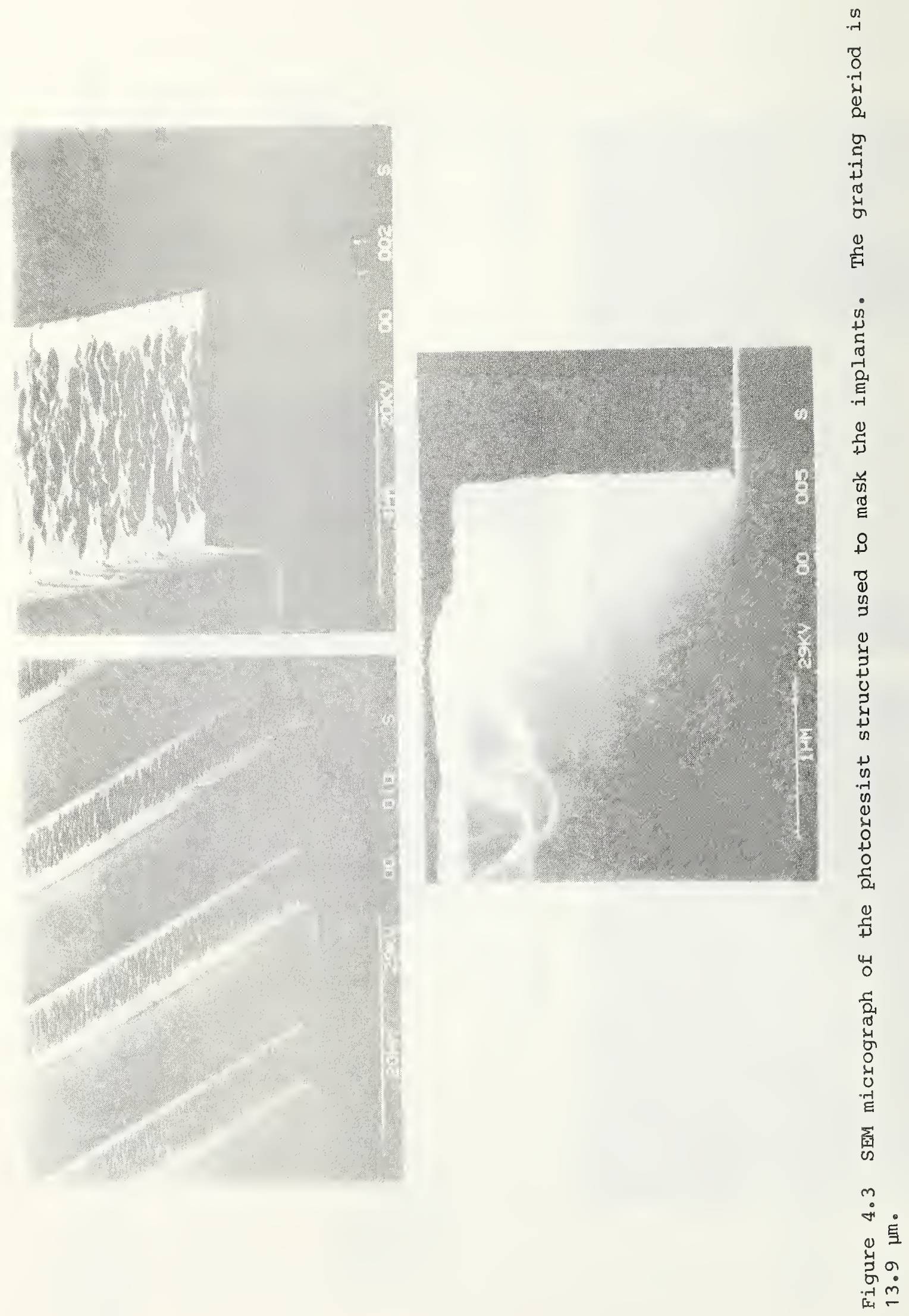


it necessary to short the top $n$-type regions to ground to prevent charqing effects. The effect of the etch is to create a roughly hemispherical ledge at the top corner of the cleaved surface. The boundary of the etched volume on the cleaved surface is determined by the junction, i.e., where the arsenic concentration from the implant is equal to the boron concentration in the substrate. The amount of etching along the top surface is determined by the dynamics of the etching process. Figure 4.4 illustrates the situation schematically and figure 4.5 is a picture of such a ledge at the corner where it meets the oxide stripe.

\section{B) Spreading Resistance}

Beveled spreading resistance measurements were made on unpatterned wafers implanted with arsenic at $75 \mathrm{keV}$ at a dose of $1 \times 10^{15} \mathrm{~cm}^{-2}$. The substrates were p-type (boron-doped) (100) silicon with resistivities of $0.3 \Omega \cdot \mathrm{cm}$ and 5 $\Omega \cdot \mathrm{cm}$ (boron concentrations of approximately $6 \times 10^{16} \mathrm{~cm}^{-3}$ and $2.7 \times 10^{15}$ $\mathrm{cm}^{-3}$, respectively). The implanted wafers were annealed for $30 \mathrm{~min}$ at $1000^{\circ} \mathrm{C}$ in nitrogen and were then beveled at an angle of approximately 4 min of arc. Probes which left a $2-\mu \mathrm{m}$ diameter footprint were used, with the loading in the range from $4 \mathrm{~g}$ to $10 \mathrm{~g}$. The step between the data points along the bevel was between $1 \mu \mathrm{m}$ and $10 \mu \mathrm{m}$. The probe-to-probe spacing was approximately 40 $\mu \mathrm{m}$.

The beveled spreading resistance data were used to provide information about the vertical profile as well as the junction depth as obtained from the location of the cusp in the data. In addition, a selective stain was used to demark the region between the implanted arsenic region ( $n$-type) and the boron-doped substrate ( $p$-type).

Table 4.1 summarizes the results of the junction location as obtained from the cusp of the spreading resistance data and as obtained from the stain technique. There are two observations which can be made. First, the location of the junction as obtained from the spreading resistance cusp is systematically sensitive to the probe load, with the junction appearing deeper for lighter loads. This is expected on the grounds that heavier loading causes a strain-induced shorting of the junction. Unfortunately, the loading is limited by noise in the voltage signal which becomes larger at light loads. For this reason the load could not be reduced below $4 \mathrm{~g}$. Second, the stain technique shows the junction to be deeper than that obtained from the spreading resistance cusp. The reason for this difference is not clear but may, in part, be due to the probe loading and, in part, to difficulties with the stain.

As indicated above, the spreading resistance measurements were also used to obtain the vertical profile of the implanted arsenic. The data were analyzed accorring to the technique due to Dickey [55]. Other methods of analysis are possible [56], which have been compared on model data [57]. It is important to emphasize that the spreading resistance technique provides a profile of the electrically active dopants. Hence, unlike the SIMS technique which measures atomic profiles, the spreading resistance technique is sensitive to the state of activation of the dopant. As a check on the resistivity profile obtained from the $R_{S p}$ technique, the sheet resistance was obtained from integration of the profile and then compared directly with that obtained from 


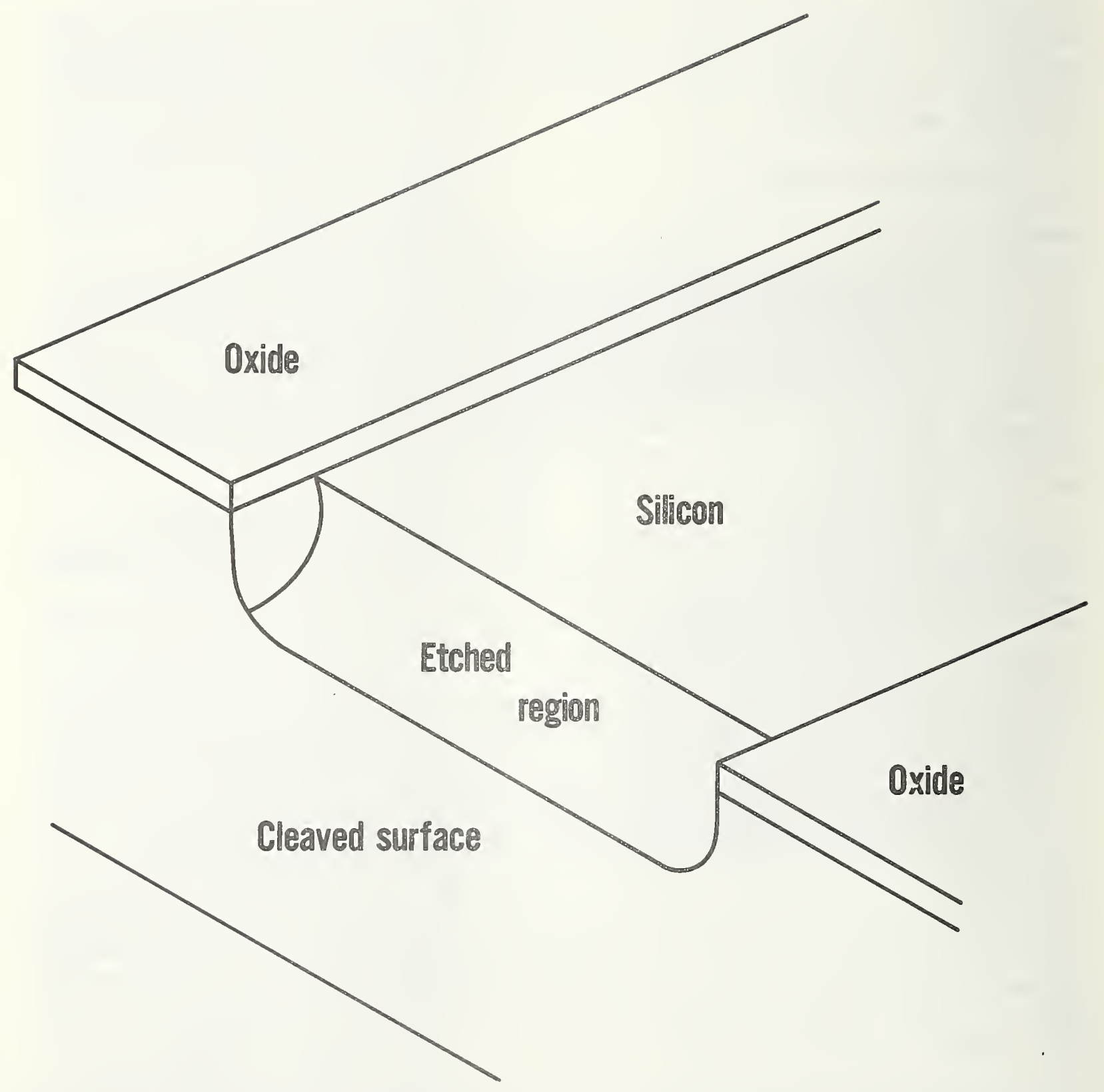

Figure 4.4 Schematic diagram of an etch step at the corner of the grating pattern. 


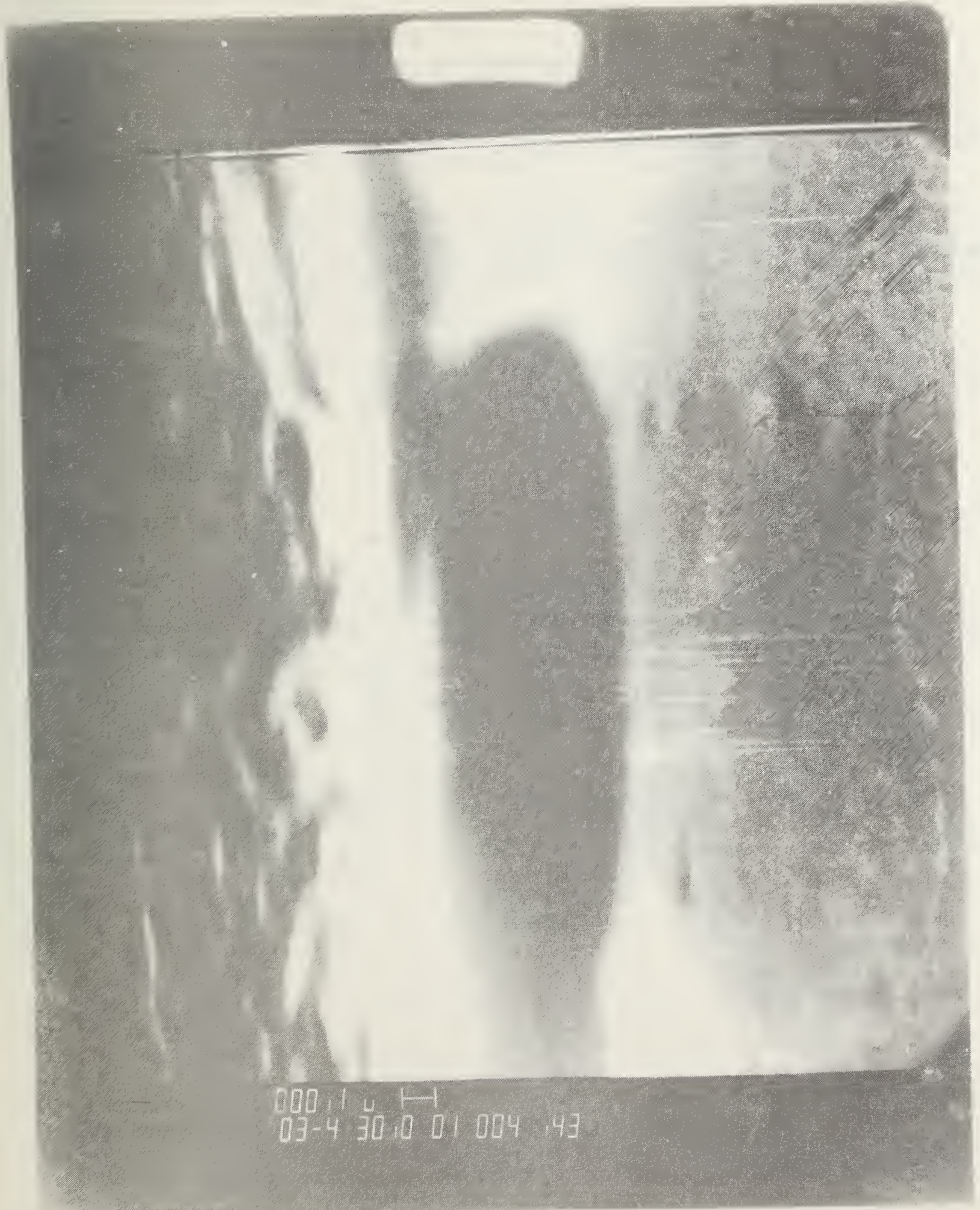

Figure 4.5 SEM micrograph of an etch step. The sample is tilted 30 deg to normal incidence. 
TABLE 4.1 - Comparison of Junction Depth Locations Obtained from Spreading Resistance and Stain Techniques.

\begin{tabular}{|c|c|c|c|c|c|c|}
\hline $\begin{array}{l}\text { Specimen } \\
\text { Substrate } \\
\text { Resistivity }\end{array}$ & $\begin{array}{l}\text { Tangent of } \\
\text { Bevel angle }\end{array}$ & $\begin{array}{l}\text { Probe } \\
\text { step ( } \mu \mathrm{m})\end{array}$ & $\begin{array}{l}\text { Probe } \\
\text { loads (gf) } \\
\text { front/rear }\end{array}$ & $\begin{array}{l}\text { Junction } \\
\text { depth from } \\
\text { cusp of } \\
\text { spreading } \\
\text { resistance } \\
\text { data }\end{array}$ & $\begin{array}{l}\text { Junction } \\
\text { depth } \\
\text { from \# } \\
\text { steps in } \\
\text { stain } \\
\text { band }\end{array}$ & $\begin{array}{l}\text { Junction depth } \\
\text { from calibrated } \\
\text { magnification } \\
\text { of stain }\end{array}$ \\
\hline $0.3 \Omega \cdot \mathrm{cm}$ & $0.0012 \pm 15 \%$ & $\begin{array}{ll}5 & \mu \mathrm{m} \\
5 & \mu \mathrm{m} \\
5 & \mu \mathrm{m} \\
5 & \mu \mathrm{m} \\
5 & \mu \mathrm{m} \\
5 & \mu \mathrm{m}\end{array}$ & $\begin{array}{l}7 / 5 \\
7 / 5 \\
7 / 5 \\
7 / 4 \\
7 / 5 \\
7 / 4\end{array}$ & $\begin{array}{l}138 \mathrm{~nm} \\
144 \mathrm{~nm} \\
150 \mathrm{~nm} \\
120 \mathrm{~nm} \\
180 \mathrm{~nm} \\
168 \mathrm{~nm}\end{array}$ & $\begin{array}{l}168 \mathrm{~nm} \\
168 \mathrm{~nm} \\
198 \mathrm{~nm} \\
198 \mathrm{~nm} \\
192 \mathrm{~nm} \\
192 \mathrm{~nm}\end{array}$ & $\begin{array}{l}- \\
- \\
- \\
- \\
-\end{array}$ \\
\hline $0.3 \Omega \cdot \mathrm{cm}$ & $0.0043 \pm 38$ & $\begin{array}{r}2.5 \\
2.5 \\
2.5 \\
5\end{array}$ & $\begin{array}{c}10 / 10 \\
6 / 6 \\
6 / 6 \\
10 / 10\end{array}$ & $\begin{array}{l}113 \mathrm{~nm} \\
183 \mathrm{~nm} \\
161 \mathrm{~nm} \\
129 \mathrm{~nm}\end{array}$ & $\begin{array}{l}161 \mathrm{~nm} \\
194 \mathrm{~nm} \\
188 \mathrm{~nm} \\
161 \mathrm{~nm}\end{array}$ & $\begin{array}{l}171 \mathrm{~nm} \\
171 \mathrm{~nm} \\
179 \mathrm{~nm} \\
179 \mathrm{~nm}\end{array}$ \\
\hline $0.3 \Omega \cdot \mathrm{cm}$ & 0.0053 & $\begin{aligned} 1 & \mu \mathrm{m} \\
2.5 & \mu \mathrm{m} \\
1 & \mu \mathrm{m} \\
1 & \mu \mathrm{m}\end{aligned}$ & $\begin{array}{l}6 / 4 \\
6 / 4 \\
6 / 4 \\
5 / 4\end{array}$ & $\begin{array}{l}160 \mathrm{~nm} \\
146 \mathrm{~nm} \\
181 \mathrm{~nm} \\
154 \mathrm{~nm}\end{array}$ & $\begin{array}{l}196 \mathrm{~nm} \\
200 \mathrm{~nm} \\
213 \mathrm{~nm} \\
218 \mathrm{~nm}\end{array}$ & $\begin{array}{l}235 \mathrm{~nm} \\
235 \mathrm{~nm} \\
235 \mathrm{~nm} \\
235 \mathrm{~nm}\end{array}$ \\
\hline $5 \Omega \cdot \mathrm{cm}$ & $\begin{array}{l}0.00092 \\
0.00092 \\
0.00101 \\
0.00111 \\
0.00111\end{array}$ & $\begin{aligned} 10 & \mu \mathrm{m} \\
10 & \mu \mathrm{m} \\
10 & \mu \mathrm{m} \\
5 & \mu \mathrm{m} \\
5 & \mu \mathrm{m}\end{aligned}$ & & $\begin{array}{l}152 \mathrm{~nm} \\
156 \mathrm{~nm} \\
162 \mathrm{~nm} \\
194 \mathrm{~nm} \\
178 \mathrm{~nm}\end{array}$ & $\begin{array}{l}193 \mathrm{~nm} \\
193 \mathrm{~nm} \\
192 \mathrm{~nm} \\
194 \mathrm{~nm} \\
189 \mathrm{~nm}\end{array}$ & $\begin{array}{l}200 \mathrm{~nm} \\
200 \mathrm{~nm} \\
197 \mathrm{~nm} \\
197 \mathrm{~nm} \\
197 \mathrm{~nm}\end{array}$ \\
\hline
\end{tabular}


the four-probe method. In the present case, a comparison of the sheet resistances shows agreement to within 20 percent. On the other hand, the dose obtained from the profile differs from the value originally used by as much as 40 to 50 percent. This difference between the integrated carrier density and the implanted ion dose is probably due to the incomplete activation problem mentioned above. In addition, the use of resistivity-dopant density relations obtained for phosphorus and applied to arsenic as well as calibration errors may be responsible for this difference. A typical spreading resistance profile as compared with the predictions of the amorphous target codes and prediction of arsenic diffusion by SUPREM [58] is presented in figure 4.6. It is important to emphasize that the amorphous tarqet codes are concerned with the distribution of the implanted ions with no predictions made about the state of activation of the dopants. The spreading resistance technique is sensitive to the resistivity variation, and hence the carrier density variation, of the implanted layer. The reason for the discrepancies remain to be determined.

\section{C) Etch Technique}

\section{i) Contrast Mechanism}

All the secondary electron pictures were taken with a beam energy of $30 \mathrm{keV}$ and a beam current of 10 to $50 \mathrm{pA}$. These are optimum conditions for hiqh resolution microscopy in our machine. Under these conditions the diameter of the electron beam spot at the surface of the sample can be approximately 0.01 $\mu \mathrm{m}$. Thirty-kilovolt electrons have a range in silicon of approximately 6.5 $\mu \mathrm{m}$ [59]. Over this range they lose energy in collisions with the electrons in the sample and scatter laterally. Contours of equal energy deposition in the silicon therefore have a characteristic "pear" shape. This situation is suggested schematically in figure 4.7 for a beam incident normal to the cleaved surface near the edge of an etched step. The secondary electron spectrum peaks below $50 \mathrm{eV}$. These low energy electrons have a range of less than $0.01 \mu \mathrm{m}$. When the beam is far from the edge, electrons excited near the top surface have enough energy to escape the silicon and be collected by the detector (a conventional phosphor-photomultiplier tube with a $+300-\mathrm{V}$ bias). As the beam is scanned near the edge of the step, electrons excited deeper in the silicon may escape laterally and be detected. Thus, as the beam moves over the step, there will be a peak in the secondary signal. When the beam is moved to the corner of the sample, there will be a large increase in the secondary signal as secondary electrons excited all along the range of the beam escape laterally. Two points are worth noting here. First, the geometry of the detector and the sample is in fact as suggested in figure 4.7. The increase in secondary electron signal at the step is therefore enhanced because the collection efficiency for electrons emitted laterally is much larger than for electrons emitted from the top surface. Second, a typical step height in this work is on the order of $0.2 \mu \mathrm{m}$. The $30-\mathrm{keV}$ beam spreads laterally very little in that distance. Therefore, for this type of sample the high resolution inherent in the electron optics can be realized.

\section{ii) Etch Data}

Figure 4.8 is a high magnification secondary electron image taken under the conditions described above. The cleaved surface is the dark area in the 


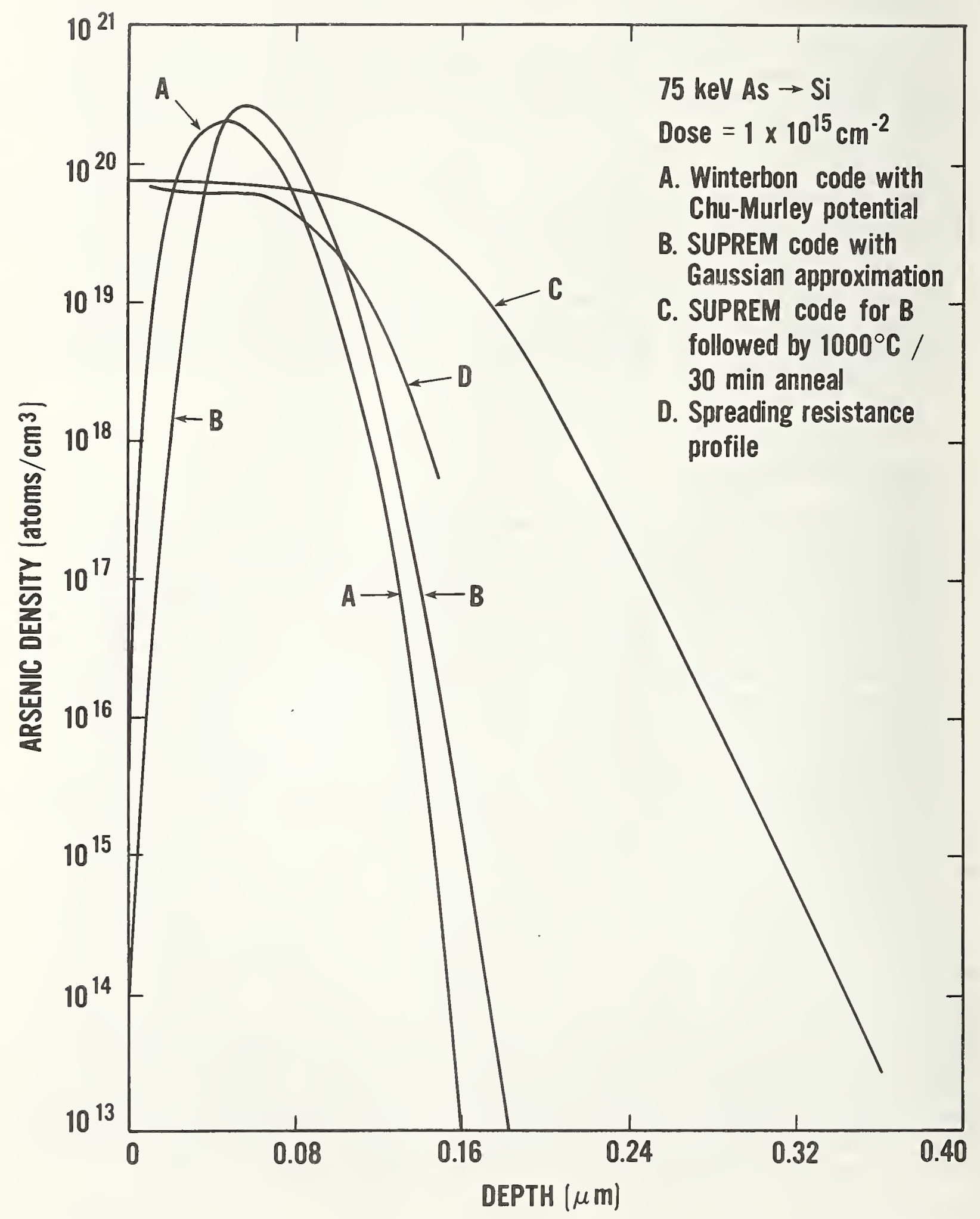

Figure 4.6 Comparison of a typical spreading resistance profile with the predictions of the SUPREM model using joined half-Gaussians and with the amorphous target codes. 


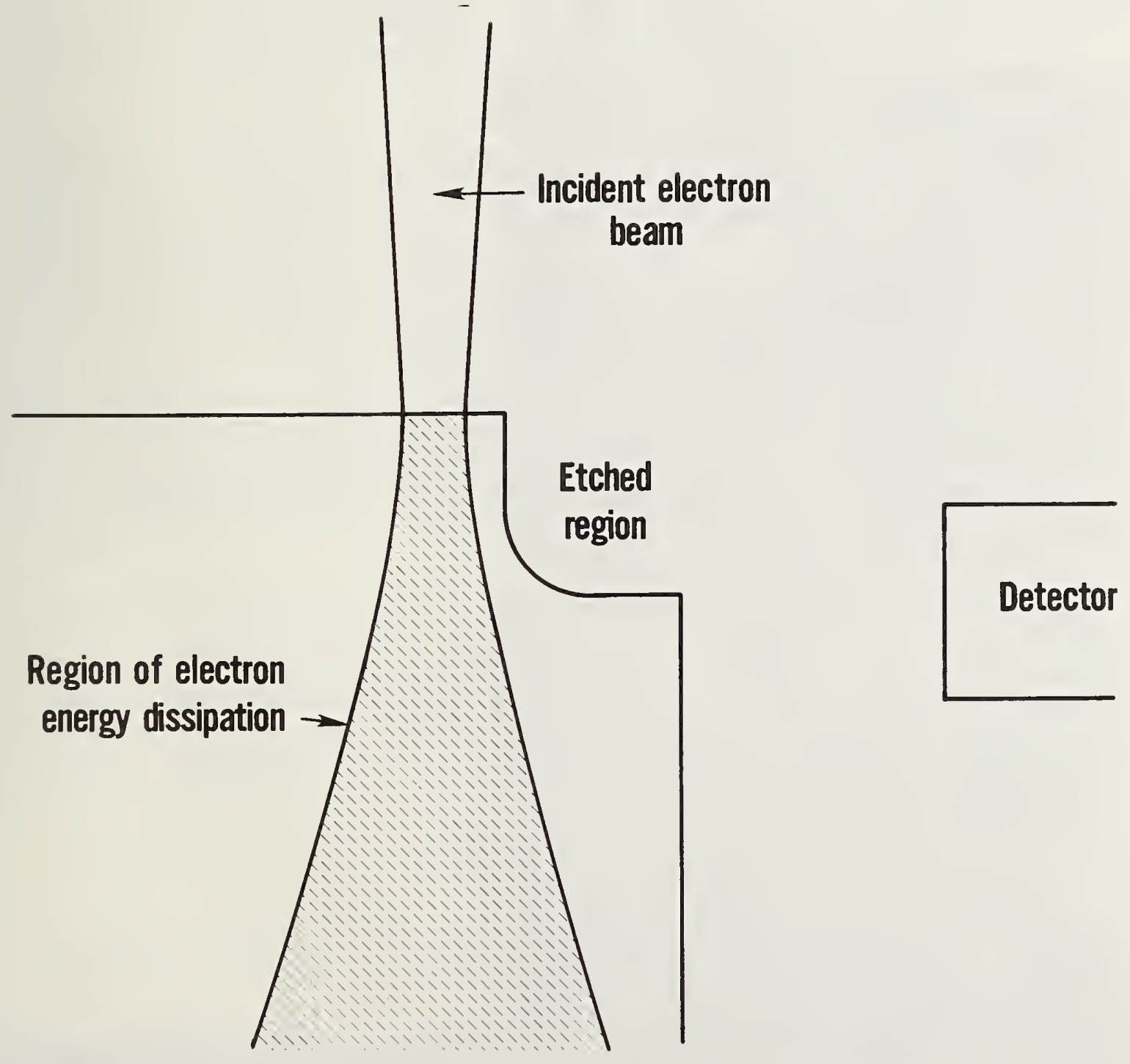

Figure 4.7 Schematic diagram of the electron-beam-sample interaction near an etch step. 


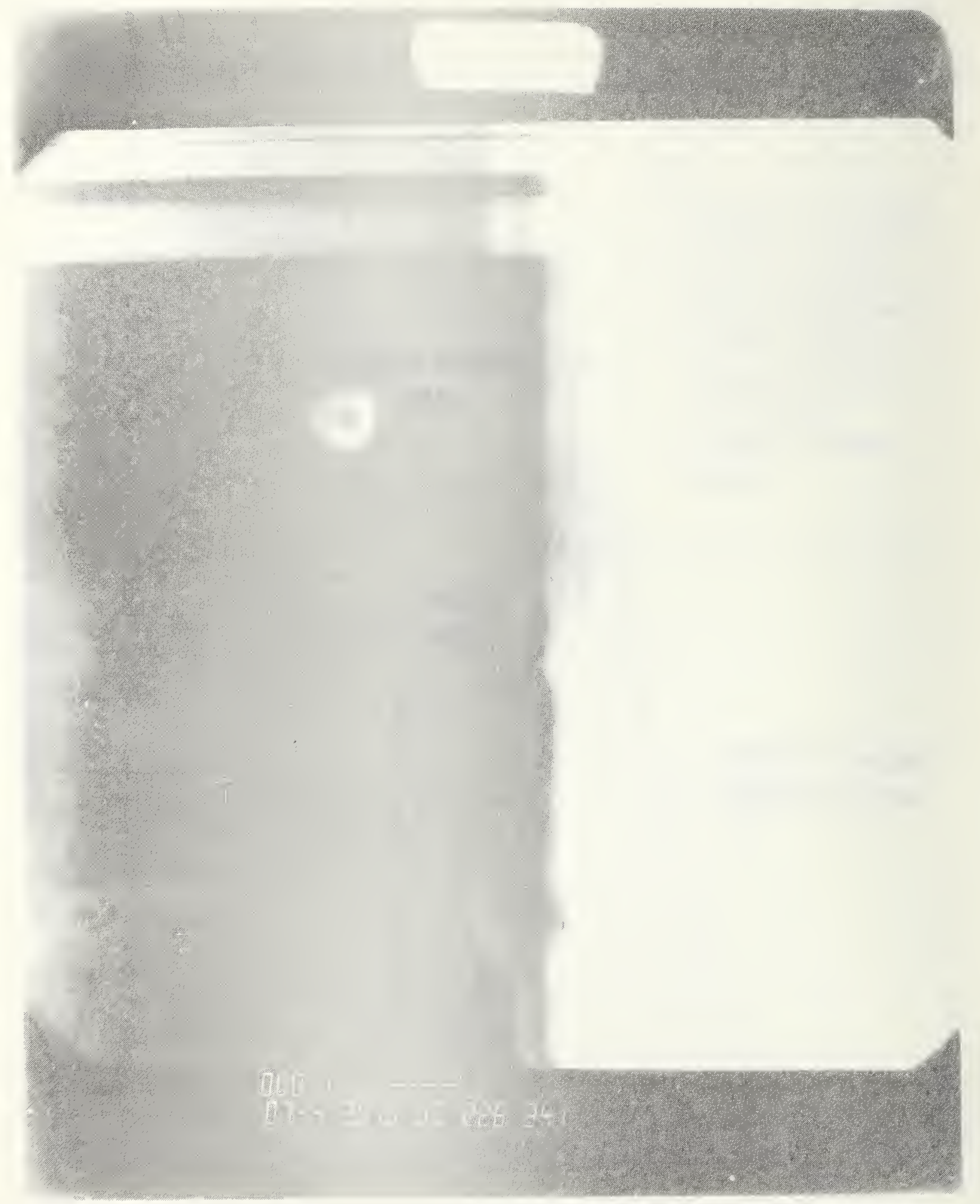

Figure 4.8 Secondary electron picture of a cleaved and etched sample tilted 0.5 deg to normal. This sample was implanted with As ${ }^{+}$to a dose of $5 \times$ $10^{15} / \mathrm{cm}^{2}$ at $75 \mathrm{keV}$ and 0 -deg tilt, then annealed at $600^{\circ} \mathrm{C}$ for $30 \mathrm{~min}$. 
lower half of the picture, the $0.05-\mu \mathrm{m}$ thick oxide stripe is in the upper left, and the etched junction area is in the center right. Several points must be noted here. The cleaved surface is actually at an angle of 89.5 deg to the incident beam. Dimensions on the cleaved area are given by the calibration bar, to within $\sim 5$ percent. The upper half of the picture, the top of the wafer, is tremendously foreshortened. The edge of the oxide therefore looks rougher than it really is, and the angle at which the stripes are tilted is exaggerated (the stripe appears to be tilted 45 deg; actually it is tilted $\sim 0.5 \mathrm{deg})$. The symmetry of the particle of dust in the lower left indicates the system is free of aberrations. Finally, the etched area is clearly delineated and the edge appears sharp to within $\sim 0.01 \mu \mathrm{m}$.

The sample in figure 4.8 was implanted at $75 \mathrm{keV}$ at 0 -deg tilt and annealed at $600^{\circ} \mathrm{C}$ for $30 \mathrm{~min}$. The junction is very shallow $(0.18 \mu \mathrm{m})$ and does not extend under the oxide. The apparent roughness of the depth of the junction region was repeated over a number of junctions. It may be due either to uneven etching or uneven activation effects at these conditions of low temperature and high concentration.

Figure 4.9 shows a similar picture, at slightly lower magnification, of a sample implanted at $150 \mathrm{keV}$ at 7 -deg tilt and annealed at $600^{\circ} \mathrm{C}$. The junction is deeper here, $0.27 \mu \mathrm{m}$, and again does not extend under the oxide. Allowing for the increased depth, we note a certain similarity of shape between the lateral profiles. In this case the junction depth is not rough, further suggesting a possible concentration-dependent problem with the previous sample.

Figure 4.10 contains two pictures, both of samples implanted at $75 \mathrm{keV}$ at 7deg tilt. In figure 4.10a, the sample was annealed at $1000^{\circ} \mathrm{C}$ for $30 \mathrm{~min}$; in figure $4.10 \mathrm{~b}$, at $1000^{\circ} \mathrm{C}$ for $60 \mathrm{~min}$. By comparison with figure 4.7 , the arsenic has clearly diffused considerably during this treatment. Between figures $4.10 \mathrm{a}$ and $4.10 \mathrm{~b}$, the depth of the junction does not change drastically, but there is considerable lateral diffusion under the oxide and the shape of the lateral profile changes.

\section{iii) Interpretation and Problems with Etch Data}

Unfortunately, after a number of other samples were examined, a systematic problem with the etch was discovered. The difficulty shows clearly in figure 4.11, which is a picture of a sample implanted at $150 \mathrm{keV}$ at 10 -deg tilt and annealed at $600^{\circ} \mathrm{C}$. The etch has proceeded in two steps. The inner, deeper region is actually what we would expect for these conditions, while the outer region is an artifact. Unfortunately, in most cases the distinction is not really clear.

Two possihle explanations are suggested for this behavior. One explanation is that, due to the high concentrations of arsenic, the small distances, and the short times involved, the arsenic is not diffusing into the etch but rather is forming a "puddle" in the etch which then redeposits on the silicon and causes further etching. The other is simply that, again due to the scales involved, the etch is simply depleting in the junction region. At this time we have not determined how to correct this problem. We note in 


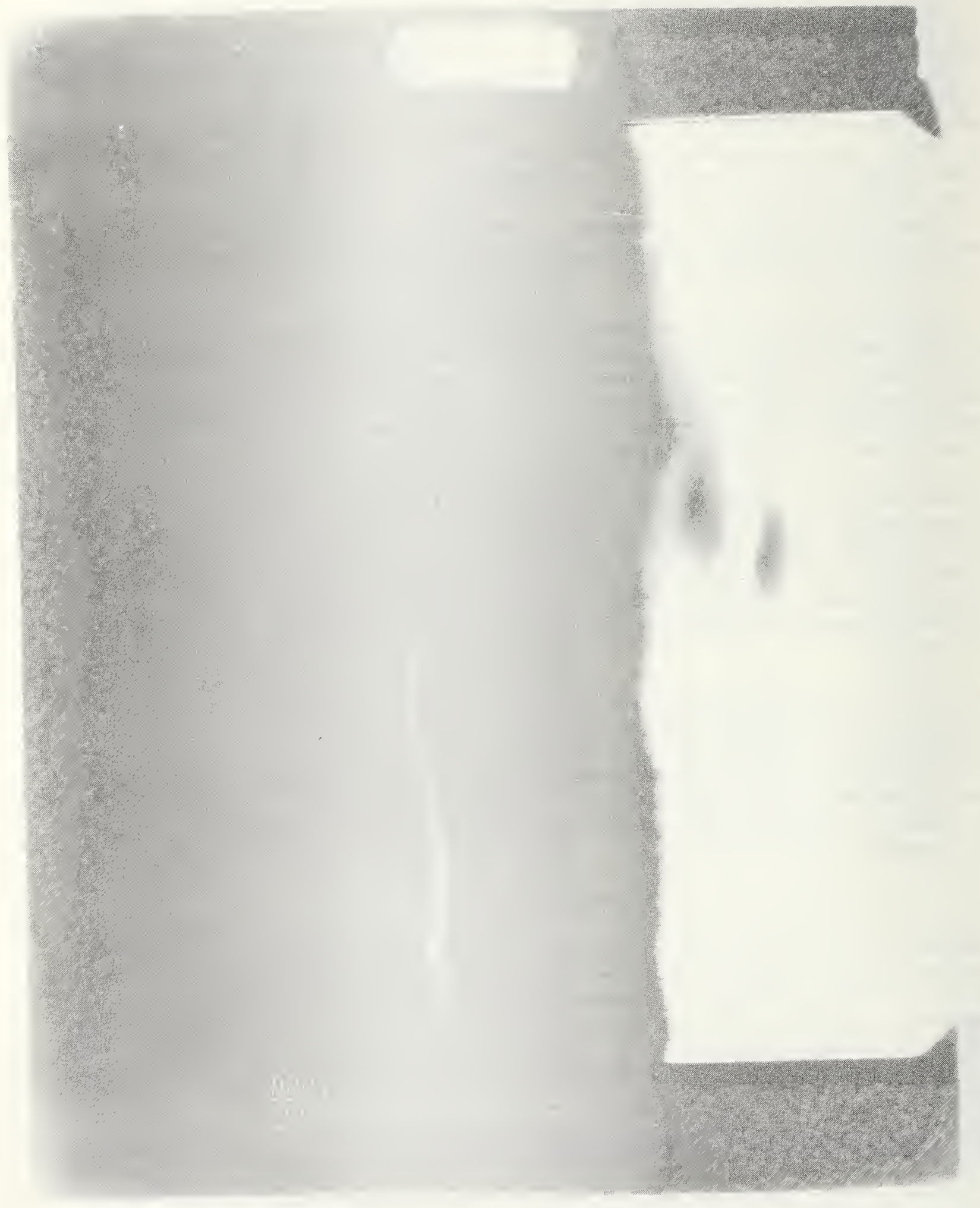

Figure 4.9 Secondary electo of a cleaved and etched sample tilted 0.5 deg to normal. This donted with $\mathrm{As}^{+}$to a dose of $5 \times$ $10^{15} / \mathrm{cm}^{2}$ at $150 \mathrm{keV}$ and $7-\mathrm{ctan}_{2}$ annealed at $600^{\circ} \mathrm{C}$ for $30 \mathrm{~min}$. 


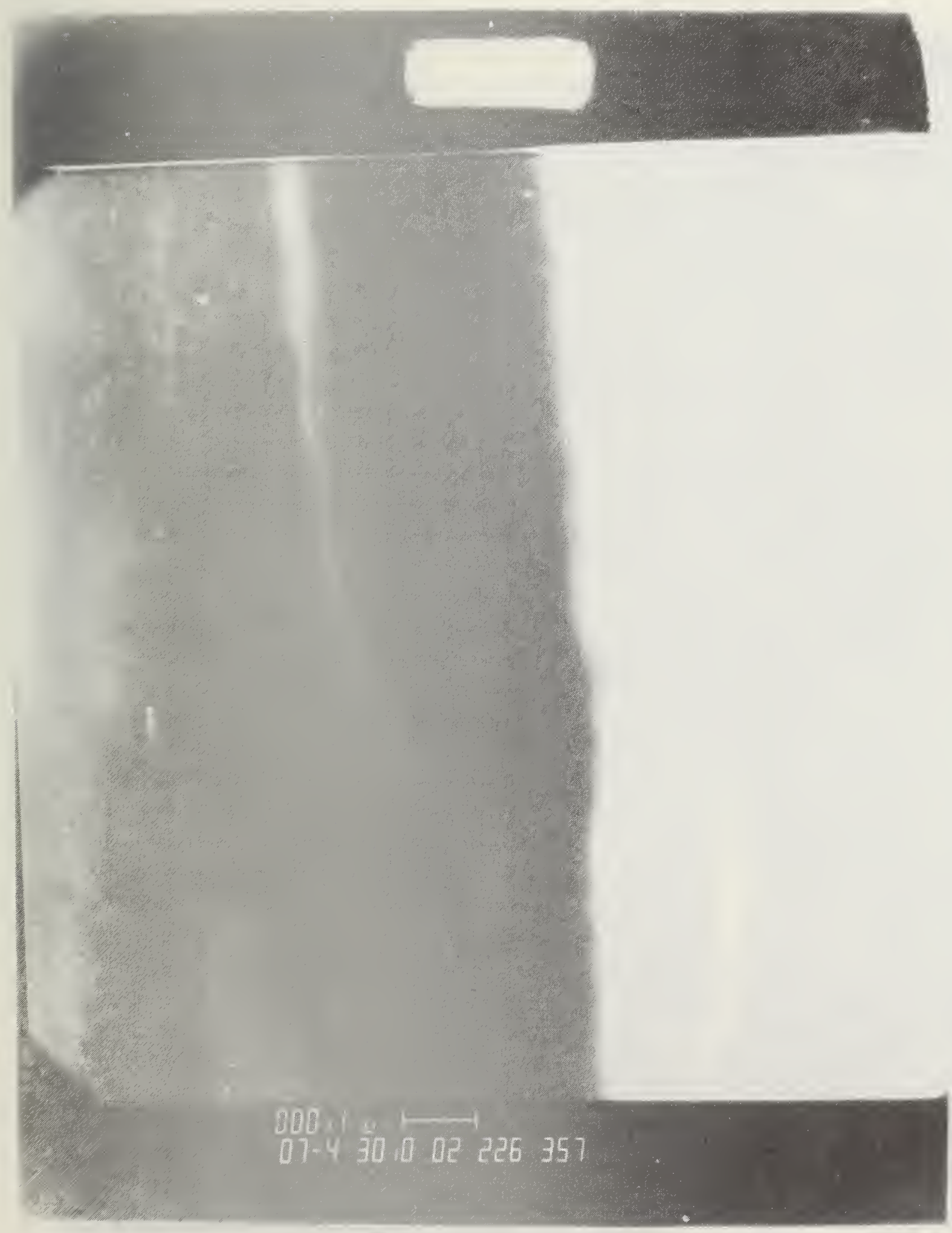

(a) Annealed at $1000^{\circ} \mathrm{C}$ for $30 \mathrm{~min}$.

Fiquxe 4.10 Secondary electron pictures of cleaved and etched samples tilted 0.5 deq to normal. Both samples were implanted with As to a dose of $5 \times$ $10^{15} / \mathrm{cm}^{2}$ at $75 \mathrm{keV}$ and 7 -deg tilt. 


$$
16
$$




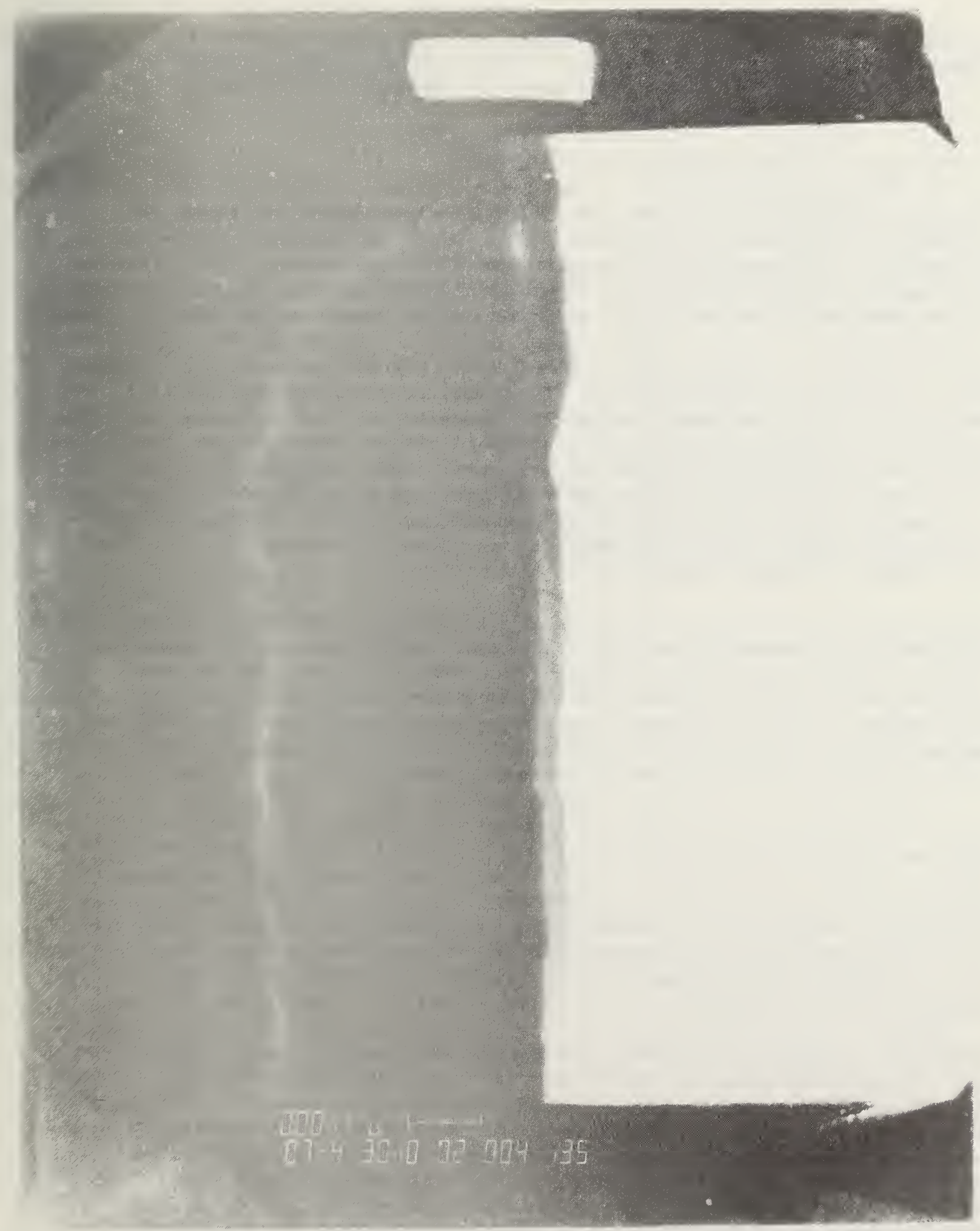

Figure 4.11 Secondary electron picture of a cleaved and etched sample 0.5 deg to normal. This sample was implanted with $\mathrm{As}^{+}$to a dose of $5 \times 10^{15} / \mathrm{cm}^{2}$ at $150 \mathrm{keV}$ and 10 -deg tilt, then annealed at $600^{\circ} \mathrm{C}$ for $30 \mathrm{~min}$. Note the doixhle step. 
closing that submicrometer etching using wet chemistry does not give uniformly good results.

\section{D) Induced Current Techniques}

\section{i) Physics of Electron-Beam-Induced Currents}

The SEM has been used to study internal fields and junctions in semiconductor devices and junctions for some time [39]. While the details of the process are complex, the underlying principles are well understood. An electron with a few tens of kilovolts of energy incident on a semiconductor will collide with the electrons and nuclei in the material and lose energy along a track of a few microns. In this study $30-\mathrm{keV}$ electrons, which have a range in $\mathrm{Si}$ of $6.5 \mathrm{~mm}$ [59], were used. The excited electrons and holes created by the initial collisions thermalize on a time scale of picoseconds, resulting in a cloud of electrons and holes around the track of the incident beam. In the absence of any electric fields, the carriers diffuse outward until, after a minority carrier lifetime, they recombine. For the beam currents used in this study, about $100 \mathrm{pA}$, plasma effects and high level injection effects do not occur. Also, for this material the minority carrier lifetime was much shorter than the time the beam dwelt on any point, so the excited carriers were in equilibrium with the beam.

The electron-beam-induced current (EBIC) apparatus consisted of a low input impedence, current-sensitive amplifier connected in series with a bias supply across a diode. In practice, contact was made to the back of the whole sample ( $p$-side) and to a large number of the n-regions on the top of the sample. Thus, a large number of diodes were connected in parallel. The resultant leakage current prohibited the use of any bias. The samples were cleaved and viewed sideways at $89.5 \mathrm{deg}$ as in the etch technique.

When the incident electron beam is directed at the $p-n$ junction, electrons and holes are created as described above. However, the built-in field at the junction causes the carriers to drift apart, electrons to the $n$-side and holes to the $p$-side, before they can recombine to result in a net current in the external amplifier. If the incident electrons are directed within a diffusion length of the junction, some of the minority carriers will diffuse to the junction and be swept across it before they recombine. Again, a net current will be observed.

The pattern of energy deposition of the incident beam affects the interpretation of the EBIC signal in two ways. Most previous work using this technique has involved more lightly doped, broader junctions in material with relatively longer lifetimes [60]. Under these conditions the lateral resolution was determined by the minority carrier lifetime and the beam scatter could be ignored. The conditions of this study involved heavily doped, highly damaged regions. Under these conditions the lateral broadening of the incident beam, which can be several tenths of a micrometer, must also be considered. The effects of lateral spread of the incident beam, diffusion length, and spatially varying dopant and damage concentration all enter the problem with comparable weight. In the other direction, the long penetration depth of the incident beam perpendicular to the cleaved surface assures that the EBIC signal will not be dominated by surface fields or surface recombination. The 
EBIC signal, therefore, is expected to be very low until the beam is scanned close to the junction. The current increases, peaks when the beam is at the junction, then steadily decreases as the beam scans away from the junction.

\section{ii) EBIC Data}

Figure 4.12 shows a composite picture taken with both secondary and EBIC signals. The sample was implanted with $\mathrm{As}^{+}$at a dose of $5 \times 10^{15} / \mathrm{cm}^{2}$ and at an energy of $75 \mathrm{keV}$ and was then annealed at $1000^{\circ} \mathrm{C}$ for $30 \mathrm{~min}$. The sample was viewed at a 0.5 -deg tilt. The oxide stripes in the top part of the picture were imaged by the secondary electrons, while the bright areas under the implanted regions were due to the EBIC signal. The EBIC signal is clearly following the junction shape.

Figure 4.13 is a higher magnification view of the sample shown in figure 4.12. The etch picture for this sample is shown in figure 4.10a. Note the change of scale. The junction actually occurs almost at the top of the wide band due to the EBIC signal. This result is to be expected. The region below the junction is unimplanted, lightly doped, undamaged p-type material. The diffusion length is long and dominates the current collection process. The region above the junction is very heavily doped, damaged n-type material. Lateral spread of the electron beam dominates the current collection, since the lifetime is very small.

Figure 4.14 is a deflection modulation picture of the EBIC signal for the sample shown in fiqure 4.13. In this mode, as the beam is scanned across the sample from left to right, the amplitude of the signal is added to the $y-$ displacement of the beam rather than modulating the intensity. The asymmetry of the signal can be seen clearly: the signal slopes off much more gradually to the left than to the riqht. The apparent uniformity of the signal in figure 4.13 is an artifact of the limited dynamic range of the photographic films.

\section{iii) Interpretation of EBIC Data}

The complexity of the EBIC signal can best be seen in figure 4.14. Several different slopes are apparent as the beam is scanned across the junction, due to different processes increasing in importance. Resolution is somewhat complicated in this context. On fairly general grounds it can be shown that the junction occurs at the peak of the signal. The location of the peak can be determined to within $100 \mathrm{~nm}$ on the linescan. Careful comparison with the secondary image gives a junction depth of $0.15 \mu \mathrm{m}$ (as compared to 0.18 for the etch).

Further interpretation of the EBIC signal will require comparison with twodimensional model programs, which will in turn require computer data acquisition of the pictures. Such an effort could, however, yield information on the spatial variation of dopants and damage which is unavailable in the etch data. 


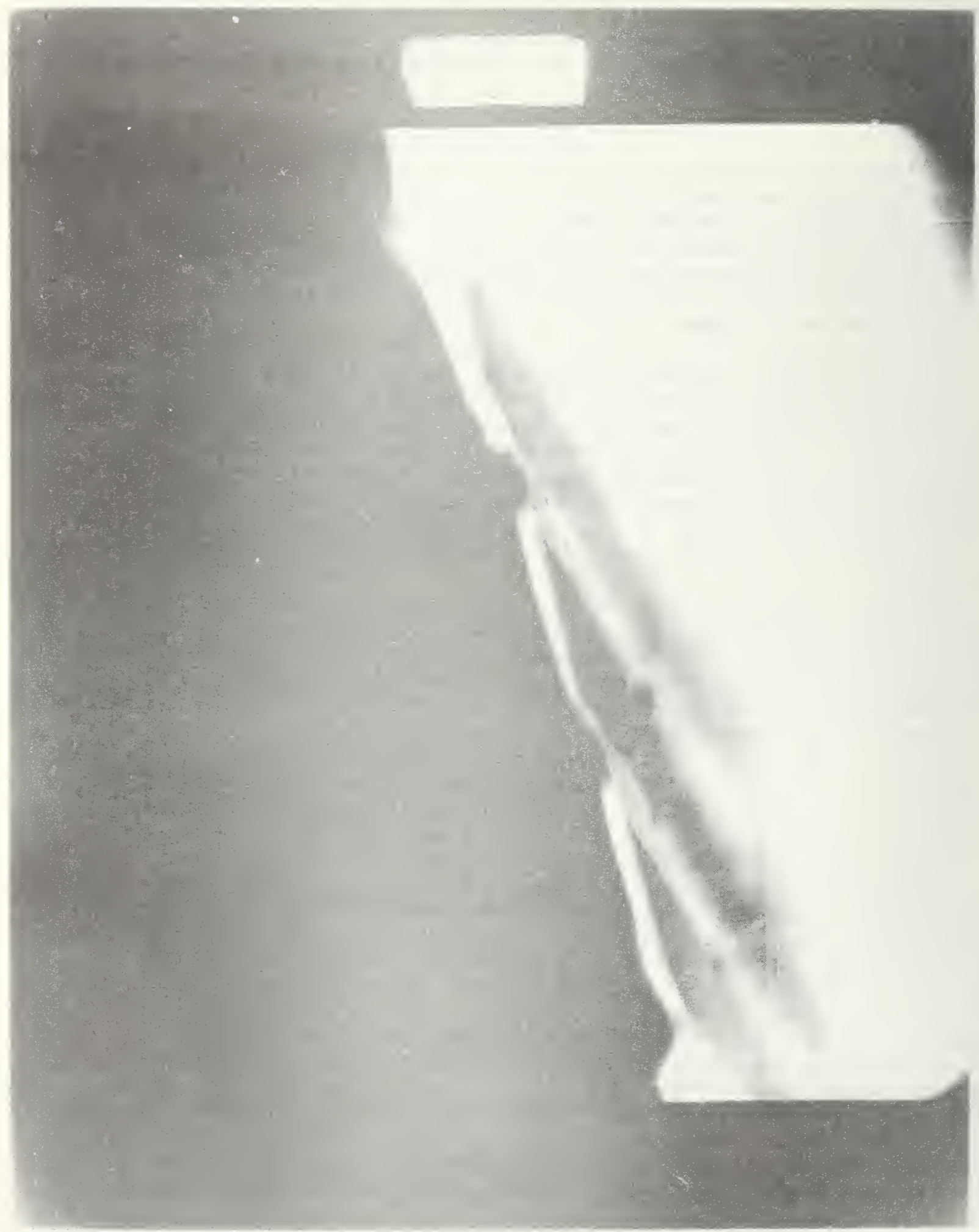

Figure 4.12 Composite secondary electron and EBIC micrograph. The sample was implanted with $\mathrm{As}^{+}$to a dose of $5 \times 10^{15} / \mathrm{cm}^{2}$ at $75 \mathrm{keV}$ and 7 -deg tilt, then annealed at $1000^{\circ} \mathrm{C}$ for $30 \mathrm{~min}$. The oxide shapes are seen in secondary electron mode, while the bright regions under the implanted states are the EBIC signal. 


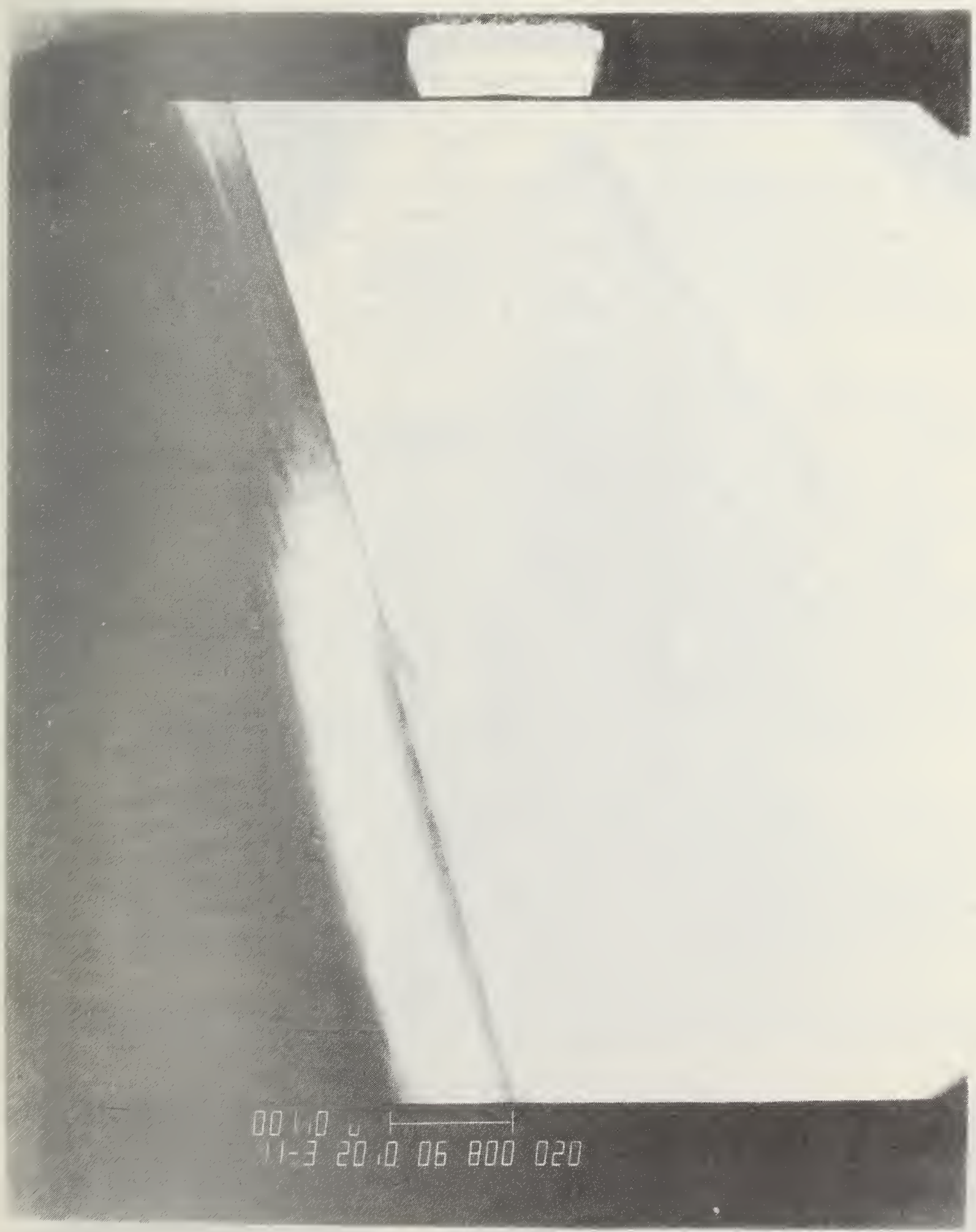

Figure 4.13 Higher magnification of figure 4.12. 


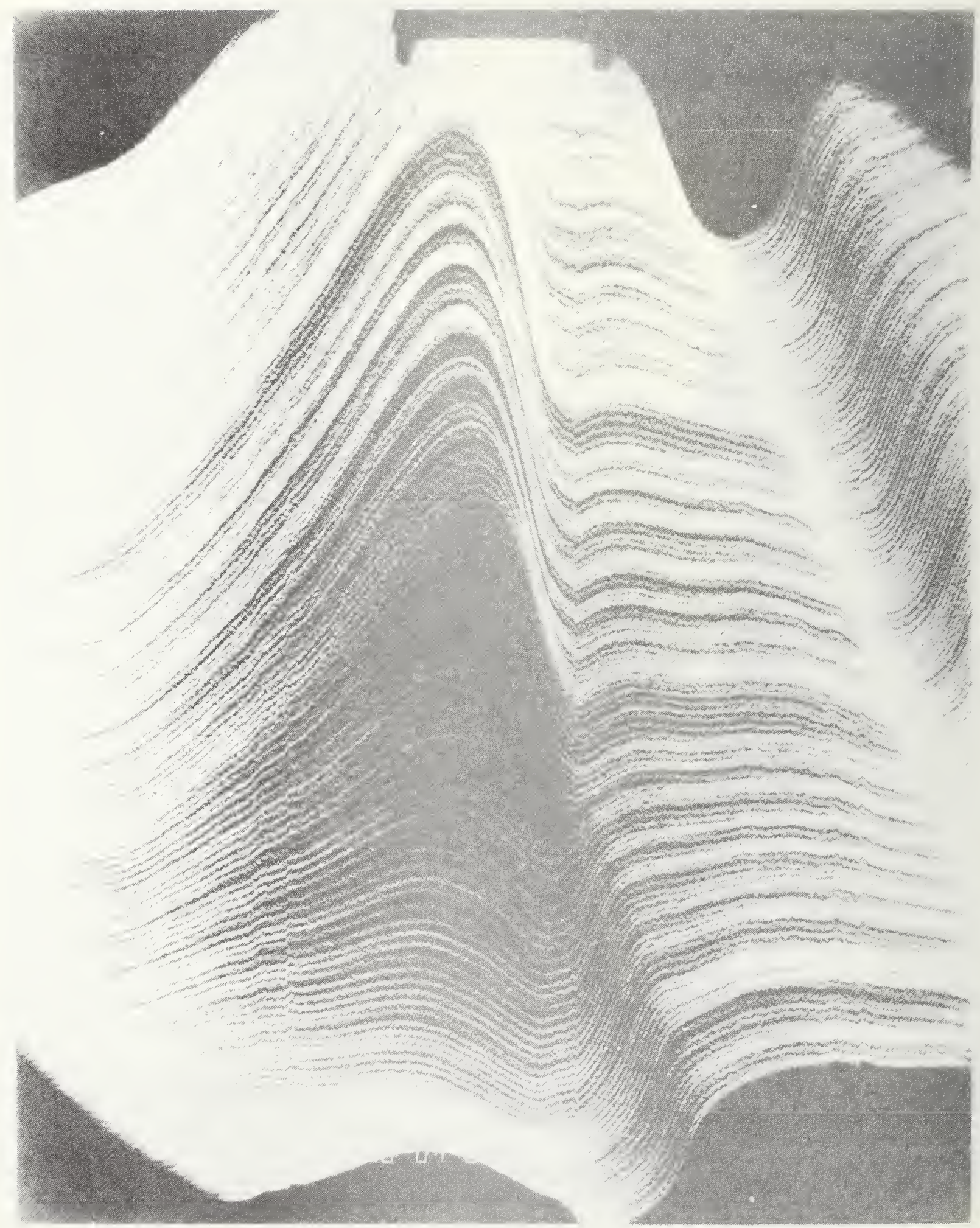

Figure 4.14 Deflection modulation EBIC picture. The sample and the geometry are the same as in figure 4.12. The asymmetry of the EBIC signal on the unimplanted and implanted sides of the junction can be seen clearly. 


\section{CONCLUSIONS}

A number of important conclusions have emerged from this study. In this section these will be noted, under the caveat that the range of conditions over which they apply has not been fully determined.

First, junction etch and EBIC techniques have both been demonstrated with submicrometer resolution. The junction etch technique has an observed edge resolution of $\sim 10 \mathrm{~nm}$; however, control of the etch process may not be that good. The EBIC technique has an observed resolution of $\sim 100 \mathrm{~nm}$; however, improved data analysis may sharpen this resolution and provide additional information. The suitability of these techniques on the scale required for VLSI and VHSIC had not previously been shown.

Second, the etch technique may be compared with existing one-dimensional theory. Figure 5.1 shows the junction depth determined by etching a sample annealed at $600^{\circ} \mathrm{C}$ as compared with the junction depth determined by the asimplanted Gaussian profile used in SUPREM. The agreement is within $10 \mathrm{~nm}$ and might be even better if there were diffusion on that scale at $600^{\circ} \mathrm{C}$. Figure 5.2 shows the same comparison for the theories discussed in chapter 3. The agreement is again within $10 \mathrm{~nm}$ and the error is about the same. High dose $\mathrm{As}^{+}$implants are nearly ideal cases for these theories, so the agreement should be taken as proof of the etch technique rather than as proof of the theories. Figure 5.3 shows a comparison of the junction depth determined by etching and by two one-dimensional diffusion models for a sample annealed at $1000^{\circ} \mathrm{C}$ in nitrogen for $30 \mathrm{~min}$. The agreement here is not within bounds, especially for SUPREM, and the deviation is in the wrong direction. Diffusion of As in $\mathrm{Si}$ at high concentrations is known to be a problem, so this result should be taken as failure of the models.

Third, the etch technique and the EBIC technique agree to within $100 \mathrm{~nm}$, the resolution of the peak in the EBIC linescan. This consistency argues well for the validity of both techniques. Spreading resistance, however, consistently indicates shallower junctions than either the etch, EBIC, or the models. Reasons for this discrepancy have been suggested and are being pursued, but the validity of submicrometer, high concentration spreading resistance is in question.

Fourth, the nonlinear diffusion of $\mathrm{As}$ in $\mathrm{Si}$ is experimentally demonstrated. Models which accurately predict this behavior, even in one dimension, do not appear to be available.

Fifth, it has been shown that implanted $\mathrm{As}^{+}$does not appreciably scatter laterally under the edge of a sharp mask. This result runs counter to some suggestions made in the past; however, this result is as expected from the previous discussion on lateral channeling. The range of validity for this conclusion must be established, and as more data become available, better models can be developed.

Sixth, the theories available for one-dimensional ion implantation and diffusion have been examined. The implantation model appears to be in good agreement but the diffusion model appears to be somewhat less accurate. The calculation of two-dimensional implant profiles has been considered and has been 


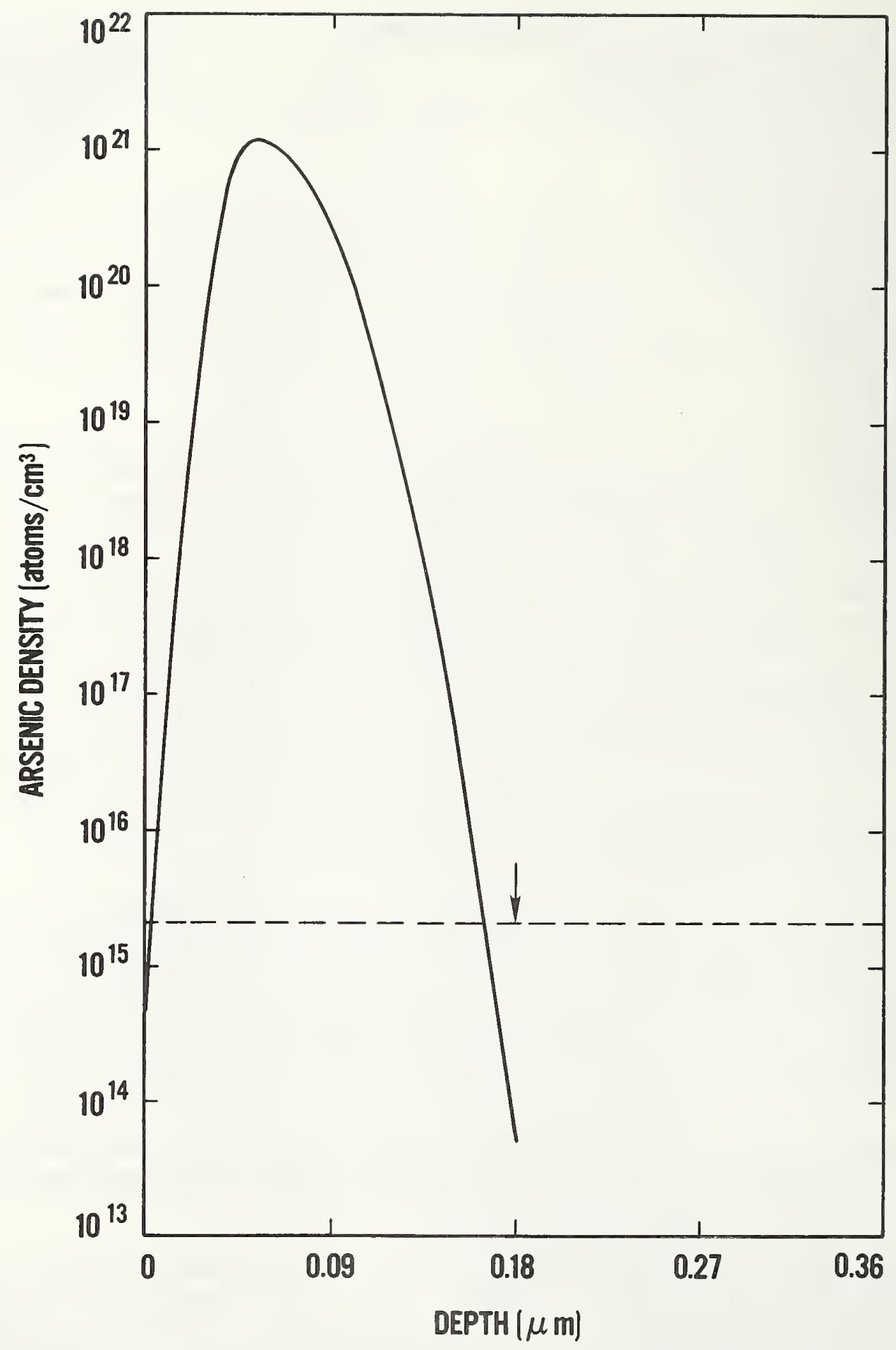

Figure 5.1 Comparison of junction depths determined by junction etch and by the as-implanted joined half-Gaussian model used in SUPREM for a sample implanted at $75 \mathrm{keV}$ and annealed at $600^{\circ} \mathrm{C}$. The line at $3 \times 10^{15}$ indicates the background, opposite conductivity-type, dopant concentration. The depth at which the Gaussian intersects this line is the predicted junction depth, while the arrow indicates the location of the junction obtained by the etch technique. 


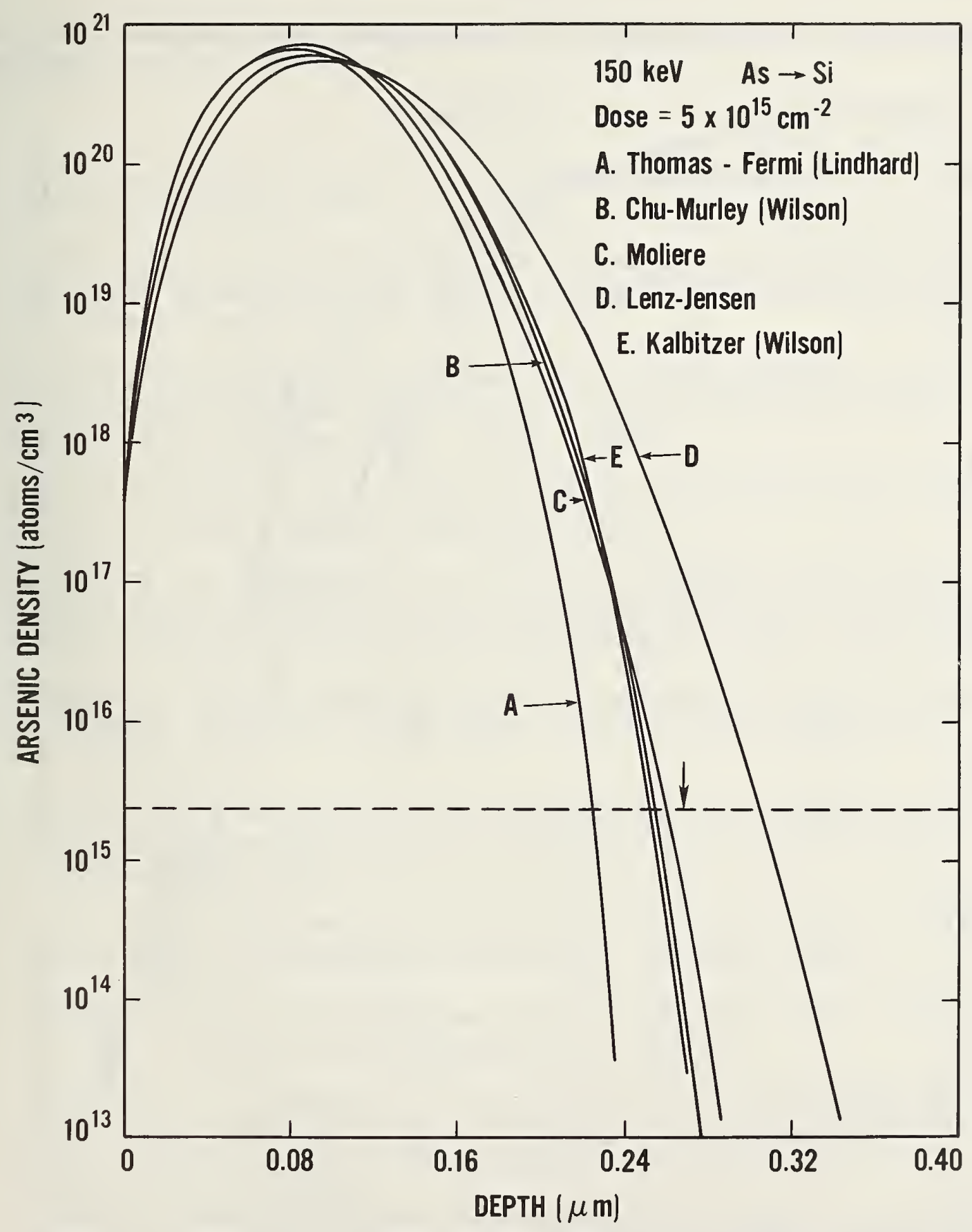

Figure 5.2 Comparison of junction depths determined by junction etch and by several theoretical models for a sample implanted at $150 \mathrm{keV}$ and annealed at $600^{\circ} \mathrm{C}$. The line at $3 \times 10^{15}$ indicates the background, opposite conductivitytype, dopant concentration. The depths at which the various curves intersect this line are the junction depths predicted by the various potential dependent models, while the arrow indicates the location of the junction as obtained by the etch technique. This figure would indicate that the chuMurley, Moliere, and Kalbitzer forms of the screened potential yield the best agreement with the observed junction depth. 


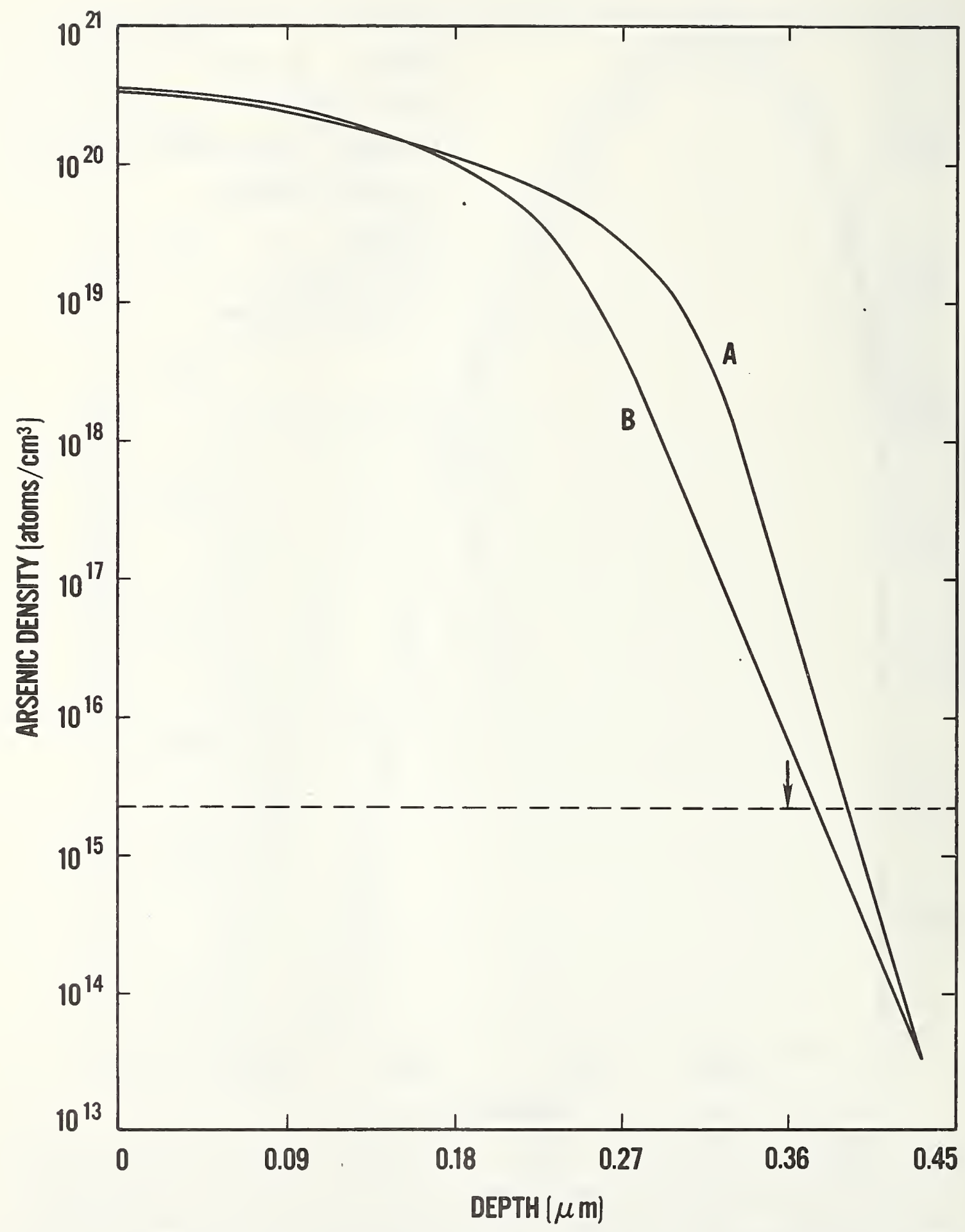

Figure 5.3 Comparison of junction depths determined by junction etch and by two models for a $5 \times 10^{15} \mathrm{~cm}^{-2} 150-\mathrm{keV}$ arsenic-implanted sample annealed at $1000^{\circ} \mathrm{C}$ for $30 \mathrm{~min}$. The horizontal line represents the background dopant concentration. The intersections of the curves with this line are the junction depths predicted by the two models for arsenic diffusion. Curve (A) is the result calculated by SUPREM II, whereas curve (B) is the result calculated by Lowney. 
shown to be in qualitative agreement with the experimentally determined twodimensional junction locations.

\section{REFERENCES}

1. Lee, D. H., and Mayer, J. W., Ion Implanted Semiconductor Devices, Proc. IEEE 62 , 1241-1255 (1974).

2. Aubuchon, K. G., The Use of Ion Implantation to Set the Threshold Voltage of MOS Transistors, Int. Conf. on Properties and Use of M.I.S. Structures, Grenoble, France, 1969, p. 575.

3. Bower, R. W., Dill, H. G., Aubuchon, K. G., and Thompson, S. A., MOS Field Effect Transistors Formed by Gate Masked Implantation, IEEE Trans. Electron Devices ED-15, 757-761 (1968).

4. Pan, E., and Fang, F. F., Lateral Spread of Ion Implanted Impurities in Silicon, J. Appl. Phys. 45, 2801-2803 (1974).

5. Dennard, R. H., Gaensslen, F. H., Yu, H. N., Rideout, V. L., Bassous, E., and LeBlanc, A. R., Design of Ion-Implanted MOSFET's with Very Small Physical Dimensions, IEEE J. Solid State Circuits SC-9, 256-268 (1974).

6. Troutman, R. R., Ion Implanted Threshold Tailoring for Insulated Gate Field-Effect Transistors, IEEE Trans. Electron Devices ED-24, 182192 (1977).

7. Rideout, V. L., Gaensslen, F. H., and LeBlanc, A. R., Device Design Considerations for Ion Implanted n-Channel MOSFET's, IBM J. Res. Dev. 1. 50-58 (January 1975).

8. Yau, I. D., A Simple Theory to Predict the Threshold Voltage of ShortChannel IGFET's, Solid-State Electron. 17, 1059-1063 (1974).

9. Troutman, R. R., VLSI Limitations from Drain-Induced Barrier Lowering, IEEE Trans. Electron Devices ED-26, 461-469 (1979).

10. Shimohigashi, K., Barnes, J. J., and Dutton, R. W., Characteristics of Short Channel MOSFET's in the Punch-Through Current Mode, Technical Digest, IEEE Int. Electron Devices Meeting, Washington, D.C., December 1978, pp. 66-69.

11. Troutman, R. R., Subthreshold Design Considerations for IGFET'S, IEEE J. Solid State Circuits sC-9, 55-60 (1974).

12. Toyabe, T., Yamaguchi, K., Asosi, S., and Mock, M. S., A Two Dimensional Avalanche Breakdown Model of Submicron MOSFET's, Technical Digest, IEEE Int. Electron Devices Meeting, Washington, D.C., December 1977, pp. $432-435$.

13. Cottre11, P. E., Troutman, R. R., and Ning, T. H., Hot Electron Emission in n-Channel IGFET's, IEEE Trans. Electron. Devices ED-26, 520-533 (1979). 
14. Liechti, C. A., Microwave Field-Effect Transistors 1976, IEEE Trans. Microwave Theory and Techniques MTT-24, 279-300 (1976).

15. Cooke, H. F., Microwave Transistors: Theory and Design, Proc. IEEE 59, 1163-1181 (1971).

16. Phillips, A. B., Transistor Engineering (McGraw Hill, New York, 1962).

17. Blood, P., Dearnaley, G., and Wilkins, M. A., The Origin of Non-Gaussian Profiles in Phosphorus-Implanted Silicon, J. Appl. Phys. 45, 51235128 (1974).

18. Lindhard, J., Influence of Crystal Lattice on Motion of Energetic Particles, K. Dan. Vidensk. Selsk. Mat. Fys. Medd. 34, No. 14 (1965).

19. Wilson, R. G., Dunlap, H. L., Jamba, D. M., and Myers, D. R., Semiconductor Measurement Technology: Angular Sensitivity of Controlled Implanted Doping Profiles, NBS Special Publication 400-49 (1978).

20. Morgan, D. V., and Van Vliet, D., Critical Approach Distances and Critical Angles for Channeling, Radiat. Eff. 8, 51-61 (1971).

21. Myers, D. R., Wilson, R. G., and Comas, J., Considerations of Ion Channeling for Semiconductor Microstructure Fabrication, J.Vac. Sci. Technol. 16, 1893-1896 (1979).

22. Inoue, $K_{0}$, Hirao, T., Yaegashi, Y., and Takayanagi, S., Asymmetrical Profiles of Ion Implanted Phosphorus in Silicon, Jap. J. Appl. Phys. $18,367-372(1979)$.

23. Myers, D. R., Comas, J., and Wilson, R. G., Effect of Silicon Dioxide Surface-Layer Thickness on Boron Profiles for Directly Aliqned Implants Into (100) Silicon, J. Appl. Phys 52, 3357-3359 (1981).

24. Desalvo, A., Galloni, R., Rosa, R., and Zignani, F., Experimental and Computer Analysis of $\mathrm{P}^{+}$-Ion Penetration Tails in a $\mathrm{SiO}_{2}-\mathrm{Si}$ Two-Layer system, J. Appl. Phys. 51, 1994-1997 (1980).

25. Klaassen, F. M•, Design and Performance of Micron-Sized Devices, SolidState Electron. 21, 565-571 (1978).

26. Sigmund, P., Collision Theory of Displacement Damage, Rev. Roun. Phys. 17, 823-870, 969-1000, and 1070-1106 (1972).

27. Firsov, O. B., A Qualitative Interpretation of the Mean Electron Excitation Energy in Atomic Collisions, J. Exper. Theoret. Phys. (USSR) 36, 1517-1523 (1959).

28. Lindhard, J., and Winther, A., Stopping Power of Electron Gas and Equipartition Rule, K. Dan. Vidensk. Mat. Fys. Medd. 34, No. 20, 1-21 (1964). 
29. Lindhard, J., Scharff, M., and Schiфtt, H. E., Range Concepts and Heavy Ion Ranges, K. Dan. Vidensk. Selsk. Mat. Fys. Medd. 33, No. 14, $1-39$ (1963).

30. Winterbon, K. B., Heavy-Ion Range Profiles and Associated Damage Distributions, Radiat. Eff. 13, 215-226 (1972).

31. Chu, W. K., Kastl, R. H., and Murley, P. C., Low Energy Antimony Implantation in Silicon, 1. Profile Measurement and Calculation, Radiat. Eff. $\underline{47}, 1-6(1980)$.

32. Wilson, W. D., Haggmark, L. G., and Biersack, J. P., Calculation of Nuclear Stopping, Ranges, and Straggling in the Low-Energy Region, Phys. Rev. B 15, 2458-2468 (1977).

33. Sanders, J. B., On Penetration Depths and Collision Cascades in Solid Materials, Thesis, Leiden (1968).

34. Hynes, J. T., and Deutch, J. M., Nonequilibrium Problems-Projection Operator Techniques, Chapter in Treatise in Physical Chemistry, Vol. VIIB, Mathematical Methods, E. Henderson, Ed. (Academic Press, New York, 1973).

35. Albers, J., and Deutch, J. M., On the Rate Equation Description of Spectral Lines, Chem. Phys. 1, 89-98 (1973).

36. Winterbon, K. B., Sigmund, P., and Sanders, J. B., Spatial Distribution of Energy Deposited by Atomic Particles in Elastic Collisions, $K$. Dan. Vidensk. Mat. Fys. Medd. 37, No. 14, 1-73 (1970).

37. Biersack, J. P., and Haggmark, L. G., A Monte Carlo Computer Program for the Transport of Energetic Ions in Amorphous Targets, Nucl. Instrum. Methods 174, 257-269 (1980).

38. Hamerseley, J. M., and Handscomb, D. C., Monte Carlo Methods (John Wiley \& Sons, New York, 1963).

39. Winterbon, K. B., Computing Moments of Implanted-Ion Range and Energy Distributions, Atomic Energy of Canada Limited Report AECL-5536 (197.6).

40. Elderton, W. P., and Johnson, N. L., Systems of Frequency Curves, pp. 35-110 (Cambridge University Press, 1969).

41. Furukawa, S., Matsumura, H., and Ishiwara, H., Theoretical Considerations on Lateral Spread of Implanted Ions, Jap. J. Appl. Phys. 11, 134-142 (1972).

42. Hofker, W. K., Implantation of Boron in Silicon, Phillips Research Reports Supplements, No. 8, pp. 1-121 (1975).

43. Gibbons, J. F., and Mylroie, S., Estimation of Impurity Profiles in IonImplanted Amorphous Targets Using Joined Half-Gaussian Distributions, Appl. Phys. Lett. 22, 568-570 (1973). 
44. Shimizu, R., Kang, S. T., Koshikawa, T., Ogata, H., Kanayama, K., Ogata, $Y_{\bullet}$, Akasaka, Y., and Harie, $K_{\bullet}$, Monte Carlo Simulation of Depth and Lateral Profiles of Boron Atoms Implanted into Polycrystalline Silicon, J. Appl. Phys. 48, 1745-1747 (1977).

45. Ryssel, H., Haberger, K॰, Hoffman, K., Prinke, G., Dumcke, R., and Sach, A., Simulation of Doping Processes, IEEE Trans. Electron Devices ED-27, 1484-1492 (1980).

46. Channeling, Theory Observation and Applications, D. V. Morgan, Ed. (Wiley, New York, 1973).

47. Gemmell, D. S., Channeling and Related Effects in the Motion of Charged Particles Through Crystals, Rev. Mod. Phys. 46, 129-227 (1974).

48. Bean, J. C., Becker, G. E., Petroff, P. M., and Seidel, T. E., Dependence of Residual Damage on Temperature During $\mathrm{Ar}^{+}$Sputter Cleaning of Silicon, J. Appl. Phys. 48, 907-913 (1977).

49. Meyer, L., Plural and Multiple Scattering of Low-Energy Heavy Particles in Solids, phys. status sol. (b) 44, 253-268 (1971).

50. Lugujjo, E., and Mayer, J. W., Energy Dependence of $\mathrm{He}^{+}$and $\mathrm{H}^{+}$Channeling in Si Overlaid with Au Films, Phys. Rev. B $7,1782-1791$ (1973).

51. Appleton, B. R., Ion Channeling Techniques for Defect and Surface Analysis, Defects in Semiconductors, J. Narayan and T. Y. Tan, Eds., pp. 97-115 (North Holland, New York, 1981).

52. Crowder, B. L., The Influence of the Amorphous Phase in Ion Distributions and Annealing Behavior of Group III and Group V Ions Implanted Into Silicon, J. Electrochem. Soc. 118, 943-947 (1971); also, Nishi, H., Sakurai, T., and Furuya, T., Electrical Activation of Implanted Arsenic in Silicon during Low Temperature Anneal, J. Electrochem. Soc. 125, 461-466 (1978).

53. Warner, D. D., and Wilson, C. L., Two Dimensional Concentration Dependent Diffusion, Bell Syst. Tech. J. 59, 1-41 (1980).

54. Kern, W., and Puotinen, D. A., Cleaning Solutions Based Hydrogen Peroxide for Use in Silicon Semiconductor Technology, RCA Rev. 31, 187206 (1970).

55. Dickey, D. H., and Ehrstein, J. R., Semiconductor Measurement Technology: Spreading Resistance Analysis for Silicon Layers with Nonuniform Resistivity, NBS Spec. Publ. 400-48 (1979).

56. D'Avanzo, D. C., Rung, R. D., Gat, A., and Dutton, R. W., High Speed Implementation and Experimental Evaluation of Multilayer Spreading Resistance, J. Electrochem. Soc. 125, 1170 (1978). 
57. Albers, J., Comparison of Spreading Resistance Correction Factor Algorithms Using Model Data, Solid-State Electronics 23, 1197-1205

(1980).

58. Autoricedes, D. A., Hansen, S. E., and Dutton, R. W., Suprem II - A Program for IC Process Modeling and Simulation, Stanford Electronics Laboratories Technical Report No. 5019-2 (June 1978).

59. Gruen, A. E., Lumineszenz-photometrische Meshugen der Energieabsorption inn Strahlungsfeld von Elektronenguellen Eindimensionaler Fall in Luft, Z. Naturforsch. 12A, 89-95 (1957).

60. Everhart, T. E., Wells, O. C., and Matta, R. K., Evaluation of Passivated Integrated Circuits Using the Scanning Electron Microscope, $J$. Electrochem. Soc. 111, 929-936 (1964). 


\begin{tabular}{|c|c|c|c|}
\hline $\begin{array}{l}\text { U.S. DEPT. OF COMM. } \\
\text { BIBLIOGRAPHIC DATA } \\
\text { SHEET (See instructions) }\end{array}$ & $\begin{array}{l}\text { 1. PUBLICATION OR } \\
\text { REPORT NO. } \\
\text { NBSIR } 81-2398\end{array}$ & 2. Performing Organ. Report No & $\begin{array}{l}\text { 3. Publication Date } \\
\text { December } 1981\end{array}$ \\
\hline \multicolumn{4}{|l|}{ 4. TITLE AND SUBTITLE } \\
\hline \multicolumn{4}{|c|}{ Investigation of the Two-Dimsional Shape of Ion-Implanted Regions } \\
\hline \multicolumn{4}{|l|}{ 5. AUTHOR(S) } \\
\hline \multirow{2}{*}{\multicolumn{3}{|c|}{$\begin{array}{l}\text { 6. PERFORMING ORGANIZATION (If joint or other than NBS, see instructions) } \\
\begin{array}{ll}\text { NATIONAL BUREAU OF STANDARDS } & \text { Sandia National Laboratories } \\
\text { DEPARTMENT OF COMMERCE } & \text { (U.S. Dept. of Energy Facility) } \\
\text { WASHINGTON, D.C. } 20234 & \text { Albuquerque, NM } 87185\end{array}\end{array}$}} & $\begin{array}{l}\text { 7MIPRtract/N6raqt-N9MP00052 } \\
\text { DE-AC04-76-DP00789 }\end{array}$ \\
\hline & & & $\begin{array}{l}\text { 8. Type of Report \& Period Covered } \\
\text { Final }\end{array}$ \\
\hline \multicolumn{4}{|c|}{ 9. SPONSORING ORGANIZATION NAME AND COMPLETE ADDRESS (Street, City, Stote, ZIP) } \\
\hline
\end{tabular}

10. SUPPLEMENTARY NOTES

[D Document describes a computer program; SF-185, FIPS Software Summary, is attached.

11. ABSTRACT (A 200-word or less factual summary of most significant information. If document includes a significant bibliogrophy or literoture survey. mention it here)

The two-dimensional shape of arsenic ion-implanted regions in single-crystal silicon was investigated both experimentally and theoretically. Experimentally, two techniques were shown to have the necessary submicron resolution: a junction etch process and an SEM-induced current collection method. A comparison of junction depths determined by the etch technique, the EBIC technique, and spreading resistance with the depths calculated using several amorphous target codes was made. For the case of low temperature $\left(600^{\circ} \mathrm{C}\right)$ anneals, the etch technique agrees very well with the junction depths predicted by the amorphous target code due to winterbon. The lateral junction locations obtained from the etch technique are in good agreement with the predictions of a two-dimensional Monte-Carlo code (TRIM) which indicates that arsenic does not show any significant lateral scattering under mask edges. For the case of high temperature $\left(1000^{\circ} \mathrm{C}\right)$ anneals, the etch and EBIC techniques agree with each other, but show consistently deeper junction locations than does the spreading resistance technique. Comparison with arsenic-diffusion models shows that concentration-dependent effects are important. Presently available processing models do not appear to adequately forecast junction depths.

12. KEY WORDS (Six to twelve entries; alphabetical order; capitalize only proper nomes; and separate key words by semicolons) Arsenic ion implantation; electron-beam-induced current; etching; integrated circuit processing; ion Ghanneling; ion implantation; lateral profiles; sGanning electron microscopy; silicon technology; spreading resistance; two-dimensional profiles.

\section{AVAILABILITY}

X] Unlimited

For Official Distribution. Do Not Release to NTIS

Order From Superintendent of Documents, U.S. Government Printing Office, Washington, D.C. 20402.

[X] Order From National, Technical Information Service (NTIS), Springfield, VA. 2216I
14. NO. OF PRINTED PAGES

71

15. Price

$\$ 8.00$ 
